\title{
Pneumonia recovery reprograms the alveolar macrophage pool
}

\author{
Antoine Guillon, ${ }^{1,2}$ Emad I. Arafa, ${ }^{1,3}$ Kimberly A. Barker, ${ }^{1,4}$ Anna C. Belkina, ${ }^{1,5,6}$ Ian Martin, ${ }^{1}$ \\ Anukul T. Shenoy, ${ }^{1}$ Alicia K. Wooten, ${ }^{1,3}$ Carolina Lyon De Ana, ${ }^{1,4}$, Anqi Dai, ${ }^{7}$ Adam Labadorf, ${ }^{7}$ \\ Jaileene Hernandez Escalante, ${ }^{4}$ Hans Dooms, ${ }^{3,4}$ Hélène Blasco, ${ }^{8}$ Katrina E. Traber, ${ }^{1,3}$ \\ Matthew R. Jones, ${ }^{1,3}$ Lee J. Quinton, ${ }^{1,3,4,5}$ and Joseph P. Mizgerd ${ }^{1,3,4,9}$ \\ ${ }^{1}$ Pulmonary Center, Boston University School of Medicine, Boston, Massachusetts, USA. ${ }^{2}$ CHRU of Tours, service de \\ Médecine Intensive Réanimation, INSERM, Centre d'Etude des Pathologies Respiratoires (CEPR), UMR 1100, University \\ of Tours, Tours, France. ${ }^{3}$ Department of Medicine, ${ }^{4}$ Department of Microbiology, ${ }^{5}$ Department of Pathology and \\ Laboratory Medicine, and ${ }^{6}$ Flow Cytometry Core Facility, Boston University School of Medicine, Boston, Massachusetts, \\ USA. ${ }^{7}$ Bioinformatics Nexus, Boston University, Boston, Massachusetts, USA. ${ }^{8}$ CHRU of Tours, Medical Pharmacology \\ Department, Inserm U1253, University of Tours, Tours, France. 'Department of Biochemistry, Boston University School of \\ Medicine, Boston, Massachusetts, USA.
}

Community-acquired pneumonia is a widespread disease with significant morbidity and mortality. Alveolar macrophages are tissue-resident lung cells that play a crucial role in innate immunity against bacteria that cause pneumonia. We hypothesized that alveolar macrophages display adaptive characteristics after resolution of bacterial pneumonia. We studied mice 1 to 6 months after self-limiting lung infections with Streptococcus pneumoniae, the most common cause of bacterial pneumonia. Alveolar macrophages, but not other myeloid cells, recovered from the lung showed long-term modifications of their surface marker phenotype. The remodeling of alveolar macrophages was (a) long-lasting (still observed 6 months after infection), (b) regionally localized (observed only in the affected lobe after lobar pneumonia), and (c) associated with macrophagedependent enhanced protection against another pneumococcal serotype. Metabolomic and transcriptomic profiling revealed that alveolar macrophages of mice that recovered from pneumonia had new baseline activities and altered responses to infection that better resembled those of adult humans. The enhanced lung protection after mild and self-limiting bacterial respiratory infections includes a profound remodeling of the alveolar macrophage pool that is long-lasting; compartmentalized; and manifest across surface receptors, metabolites, and both resting and stimulated transcriptomes.

Authorship note: $A G$ and EIA contributed equally to this work.

Conflict of interest: The authors have declared that no conflict of interest exists.

Copyright: (c) 2020, American Society for Clinical Investigation.

Submitted: August 27, 2019

Accepted: January 22, 2020

Published: February 27, 2020.

Reference information: JCI Insight. 2020;5(4):e133042.

https://doi.org/10.1172/jci.

insight.133042.

\section{Introduction}

Although respiration is the main function of the lung, its role as an immune organ is essential to health and homeostasis. The lung is under continual pressure from the omnipresent but dynamic microbiome of the respiratory tract (1). Ineffective control of microbes in the lower airways leads to dysbiosis and pneumonia, particularly in younger and older individuals (2-5). Between these ends of the age spectrum, healthy young adults have a much lower incidence and severity of pneumonia. Identifying mechanisms protecting healthy young adult lungs against pneumonia is a pressing research priority (6).

Lung protection depends on an immunological tone that microbial exposures establish (3). This dictates outcomes for subsequent infections and is as important as or more important than species or genome when using animal models to study human health and disease (7-9). Lymphocytes become long-term residents in the lung after resolution of respiratory infection, including resident memory $\mathrm{T}$ (Trm) cells, Brm cells, and others, helping improve pulmonary defense (3, 10-12). How else lungs change after resolution of infection is less clear.

Alveolar macrophages are constituent resident immune cells of the lung, present before infections occur. Although initially derived from embryonic precursors, a continuously expanding fraction is derived from monocytes instead of embryonic precursors, equilibrating around 1 year of age in mice $(13,14)$. This process is accelerated by infection, where alveolar macrophages often change in number acutely but return to starting values 
A

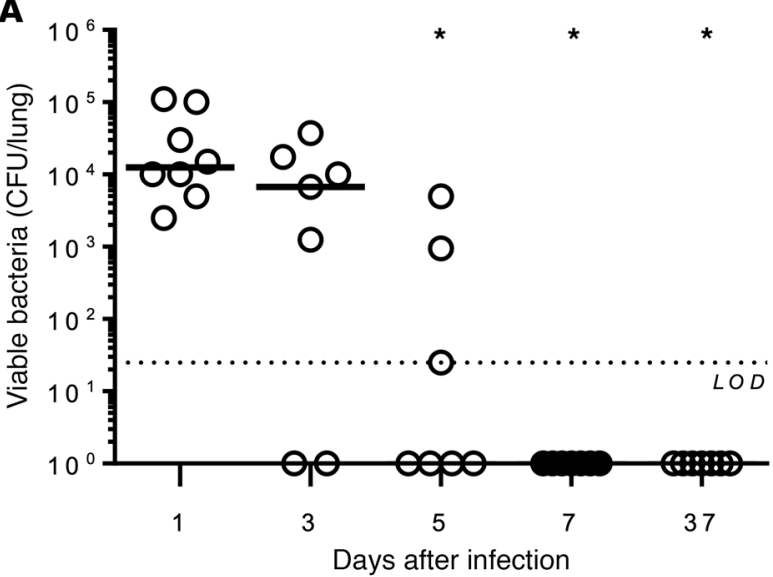

C

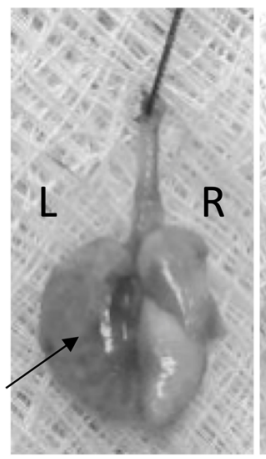

Day 1

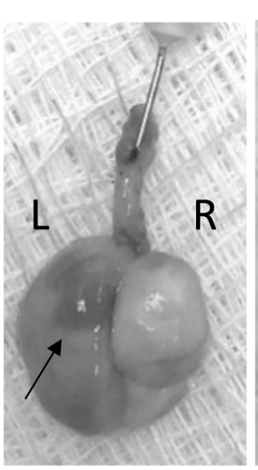

Day 3

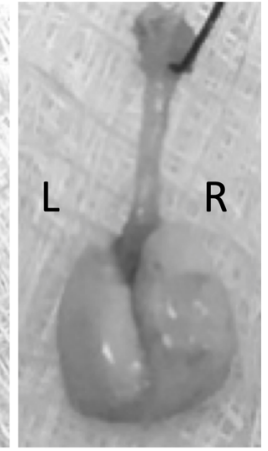

Day 7
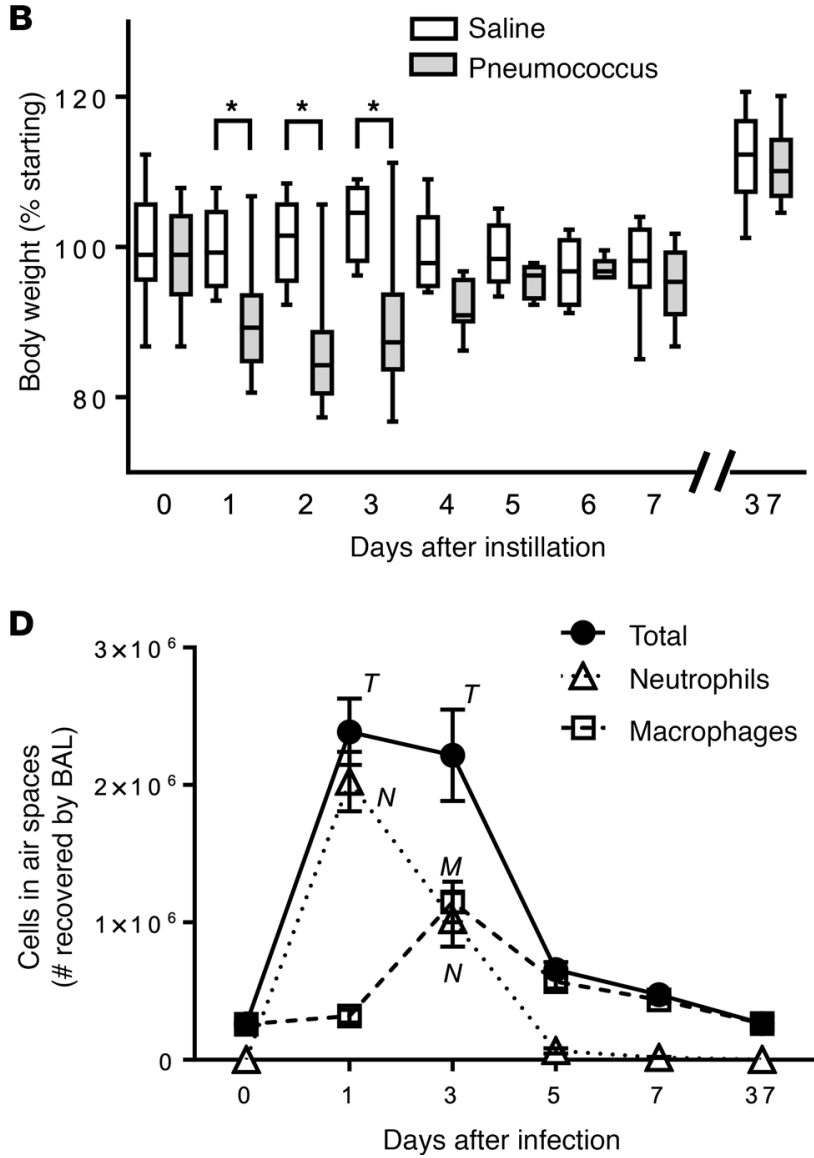

Figure 1. Murine model of self-limiting pneumonia. To determine effects of acute pneumonia, mice were infected with $10^{6} \mathrm{CFU}$ of serotype $19 \mathrm{~F}$ instilled into the left lung lobe on day 0 . To establish heterotypic immune protection, mice received a second infection with $10^{6}$ CFU of serotype $19 F$ instilled into the left lung lobe on day 7 and then were rested for 30 more days before lungs were examined. Sterile saline was used as control. Infected mice and controls were compared for (A) bacterial burden, (B) body weight, (C) gross morphology of lungs, and (D) leukocyte count in BAL, enumerated using a LUNA automated cell counter followed by a differential determined morphologically from cytocentrifuge preparations of BAL cells. In A, every individual symbol represents a single mouse, with horizontal lines showing median values for the group, and asterisks (*) indicate statistically significant $(P<0.05)$ differences from day 1 values using the Kruskal-Wallis test and Dunn's post hoc multiple-comparisons test. In B, box and whisker plots show median, quartiles, and range, with asterisks $\left(^{*}\right)$ indicating significance $(P<0.05)$ of difference between saline and pneumococcus groups as determined using 2-way ANOVA and Holm-Šídák post hoc multiple-comparisons tests. In D, summary data show mean and SEM, with significance $(P<0.05)$ of difference from day 0 determined using 2-way ANOVA and Holm-Šídák post hoc multiple-comparisons test and communicated with letters representing cell type ( $T$, total; $N$, neutrophils; $M$, macrophages). For B and D, pooled data represent a total $n$ of $5-12$ mice per group. For all, 2 independent experiments were performed. LOD, limit of detection.

after resolution $(15,16)$. The postresolution alveolar macrophage population can include a larger fraction of cells that are monocyte derived $(17,18)$. Whether alveolar macrophages of disparate origins differ functionally is uncertain. In noninflamed lungs, the embryo-derived and monocyte-derived alveolar macrophages appear largely similar to each other, including their transcriptomes and responses to microbes $(17,19)$, suggesting that environmental cues rather than cellular origins drive the alveolar macrophage phenotype.

Infection-specific cues influence macrophage phenotypes through "trained immunity" (20). The type, dose, and duration of microbial stimuli can guide monocytes and macrophages toward new antiinflammatory, proinflammatory, or other more complex phenotypes (21). Metabolic changes contribute to this training (22), with macrophage metabolism changes guided by diverse types of stimuli (such as different microbes and microbial products), tissue environments (such as different organs and their state of health or disease), and cell types (such as monocytes or macrophages or subsets thereof). There is scant information regarding trained immunity and lung infections. After mice recover from influenza infection, their alveolar macrophages exhibit a prolonged tolerance-like phenotype with decreased induction of the chemokines CXCL1 and CXCL2 in response to bacterial flagellin (15). After respiratory infection of mice with an adenoviral vaccine vector expressing a mycobacterial protein, alveolar macrophages show differences in surface marker 
expression, metabolites, and transcriptomes in the resting state, and their responses to subsequent bacterial stimulations are altered to be hyperinflammatory, including increased induction of the chemokines CXCL1 and CXCL2 (23). This pair of studies reveals a potential for extensive and profound changes in alveolar macrophage phenotypes due to prior experience with microbial stimuli, with opposing responses in the sole parameter similarly measured (chemokine induction), revealing that context may be critical.

Pneumococcus is a paramount context for the respiratory tract. All children experience multiple rounds of respiratory tract infection with pneumococcus during their first years of life, and such infections occur repeatedly throughout life (24). As a result, adult humans routinely possess multiple types of adaptive immune memory against pneumococcus (24). Despite this, pneumococcus is the most common bacterial cause of pneumonia (25-27). Although infections with pneumococcus are a hallmark human experience, and alveolar macrophage death is an important component of pneumococcal pneumonia (28-30), the effects of resolved respiratory infection by pneumococcus (or any bacterial pathogen) on alveolar macrophage phenotypes remain unexamined. Although recovery from infections alters the blood cells and lung lymphocytes of mice to be more similar to those of young adult humans $(3,8)$, alveolar macrophage remodeling after microbial exposures has yet to be connected to any human context. Here, we begin addressing these knowledge gaps in lung immunology.

\section{Results}

Murine model of resolving pneumonia. We examined the time course of infection, morbidity, and inflammation after lobar pneumonia caused by a pneumococcus serotype $19 \mathrm{~F}$ isolate, which causes a self-limiting infection in mice (31). Most mice eliminated living Streptococcus pneumoniae by 5 days after infection and all by 7 days (Figure 1A). Body weight decreased over the first 3 days compared with control mice, but differences between groups disappeared in under a week (Figure 1B). Gross morphology revealed that excised lungs had hepatization typical of lobar pneumonia at 1 and 3 days, which resolved before day 7 (Fig ure 1C). Pulmonary inflammation was measured by quantifying leukocytes in the bronchoalveolar lavage (BAL). Concomitant with the weight loss and lung hepatization, we observed increases in neutrophils (by day 1) and macrophages (by day 3) in the airspaces (Figure 1D). BAL leukocytes returned to baseline by day 5 (Figure 1D). Thus, this serotype $19 \mathrm{~F}$ isolate causes a mild and self-limiting infection in mice. A second infection is needed to establish heterotypic immunological memory effective against mismatched serotypes (11). We delivered this second infection on day 7, after mice had returned to baseline. More than 4 weeks from this second infection (day 37), mice remained free of infection (Figure 1A) and morbidity (Figure 1B). The leukocytes in these lungs will be considered further below. Mice that recovered from the initial 2 rounds of infection and were studied after at least 4 weeks from the last infection will hereafter be referred to as "experienced," to be compared with negative control mice that received sterile saline instead of pneumococcus, hereafter referred to as "naive."

Experienced lungs do not differ in numbers of myeloid cells. The lungs of experienced mice had an improved defense against an unrelated serotype of pneumococcus (Figure 2A). These lungs contain new populations of cells, namely an increase in $\mathrm{CD}^{+}$Trm cells (11). We considered whether there might also be increased populations of select myeloid cell subsets in such lungs. To differentiate and enumerate the myeloid cell populations in lung single-cell suspensions from experienced and naive mice, we used a systematic approach based on flow cytometry and sequential gating (Figure 2B) (32). We excluded debris, doublets, and dead cells and restricted analyses to $\mathrm{CD} 45^{+}$leukocytes. Alveolar macrophages were identified as $\mathrm{CD} 11 \mathrm{c}^{+}$SiglecF ${ }^{+} \mathrm{CD}^{+} 4^{+}$, which includes alveolar macrophages whether of embryonic or monocytic origin. The $\mathrm{CD} 11 \mathrm{c}^{+}$SiglecF-MHC $\mathrm{II}^{+} \mathrm{CD} 64^{+}$cells were defined as interstitial macrophages (IMs), $\mathrm{CD}_{11} \mathrm{c}^{+}$SiglecF-MHC II-Ly6C $\mathrm{C}^{+}$cells as plasmacytoid dendritic cells (pDCs), CD11 $\mathrm{c}^{+}$SiglecF-MHC $\mathrm{II}^{+} \mathrm{Ly}^{-} \mathrm{C}^{-}$cells as conventional dendritic cells (cDCs), CD11c Ly6G ${ }^{\text {bright }}$ cells as neutrophils, CD11c-SiglecF ${ }^{+}$cells as eosinophils, and CD11 ${ }^{-}$Ly6G-SiglecF-Ly6C ${ }^{\text {bright }}$ cells as monocytes. Lymphocytes would not be discriminated using this gating strategy and may account for undefined events in these panels, such as the many $\mathrm{CD}^{2} 5^{+}$SiglecF- ${ }^{-} \mathrm{CD} 11 \mathrm{c}^{-} \mathrm{Ly} 6 \mathrm{G}^{-} \mathrm{Ly}^{-} \mathrm{C}^{-}$cells. For all 7 of the myeloid cell subsets, numbers per lung were similar in experienced and naive mice (Figure 2C). One month after recovery from mild and self-limiting respiratory infections, experienced mice did not differ from naive counterparts regarding the quantities of these populations of myeloid cells in the lung.

Alveolar macrophages display a distinct phenotype in experienced lungs. Our flow cytometry panel was designed to bin cells into preassigned categories so that numbers per lung could be calculated for each cell type. Unbiased approaches to simultaneously assessing multiple markers across all immune cells may 
A

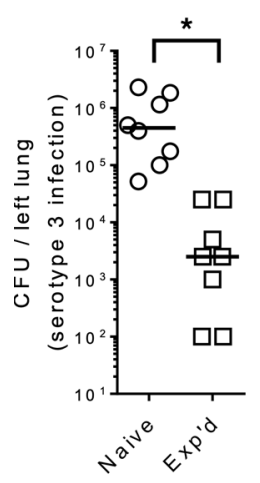

B
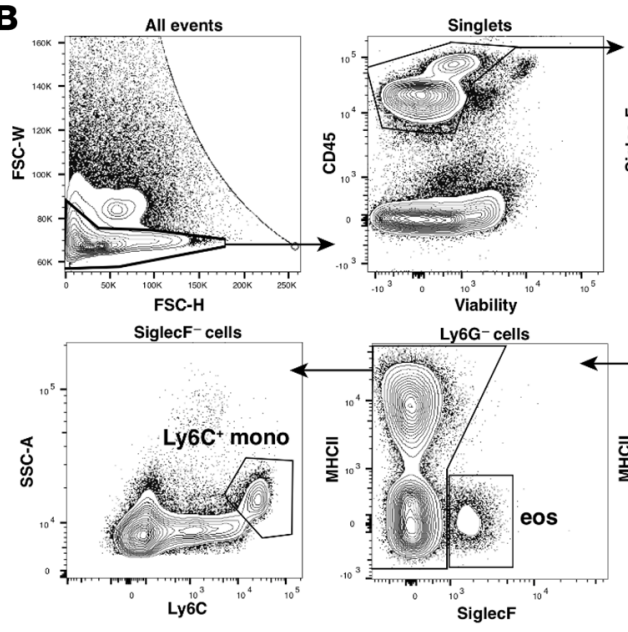

C
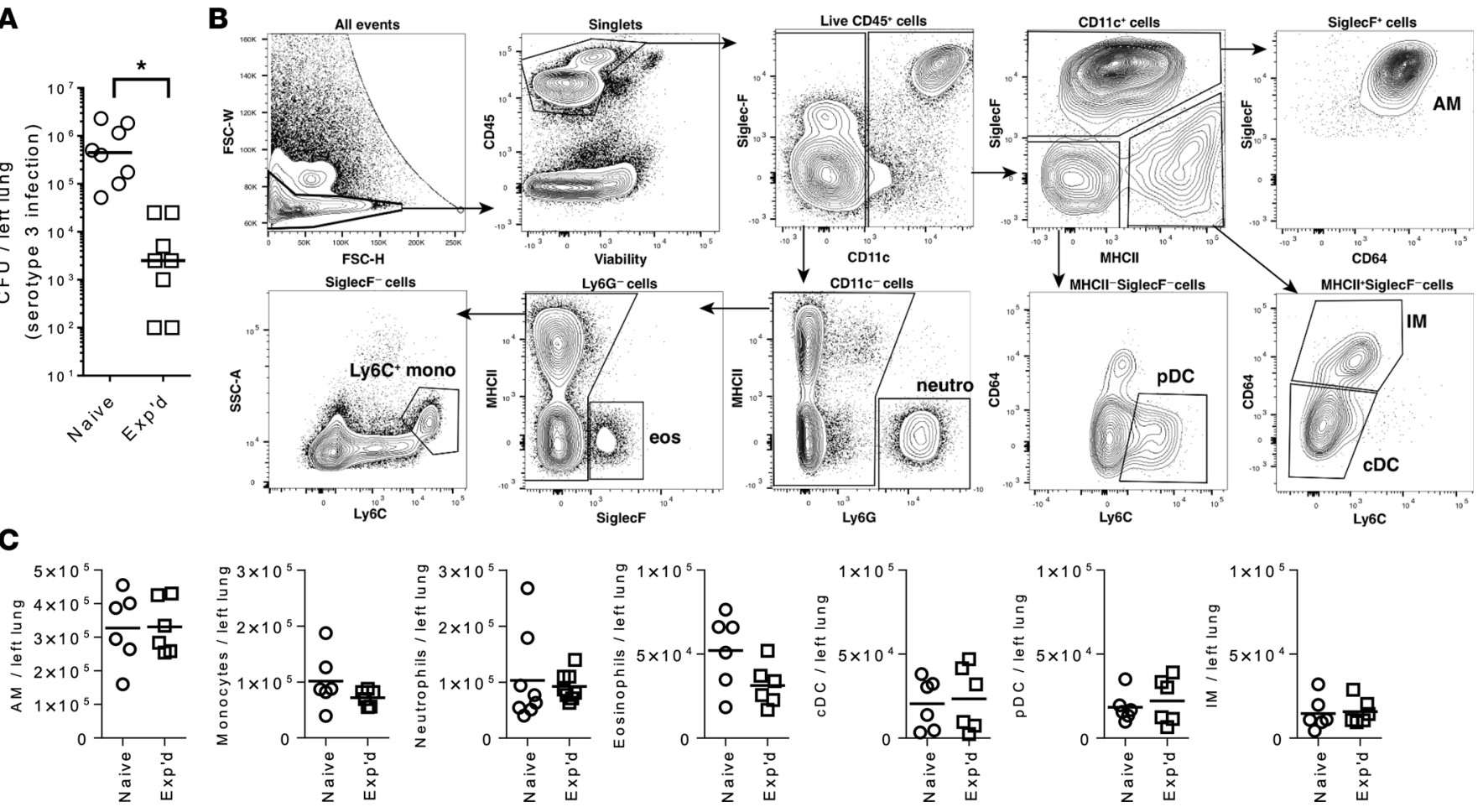

Figure 2. Immunophenotyping of lung myeloid cells 1 month after recovery from mild pneumonia. (A) Defense against a serotype 3 pneumococcal pneumonia in naive mice and experienced (Exp'd) mice with a history of serotype 19F pneumococcal infections as described in Figure 1. Naive or experienced mice were infected intranasally (i.n.) with $0.5 \times 10^{6}$ to $1 \times 10^{6} \mathrm{CFU}$, and lung bacterial burdens were determined after 24 hours of infection. Asterisk (*) indicates statistically significant $(P<0.05)$ difference between groups using Mann-Whitney $U$ test. For $\mathbf{B}$ and $\mathbf{C}$, mice received intratracheally (i.t.) left lobe serotype 19F infections (Exp'd) or saline instillations (Naive) on days 0 and 7, before left lung lobes were collected at day 37 with no further infections. (B) Gating strategy for myeloid cells in lung single-cell suspensions. Cells were isolated from enzymatically digested mouse lungs, and after the exclusion of doublets, debris, and dead cells, immune cells were identified by CD45 staining. Sequential gating strategy was used to identify myeloid cell subsets. Representative sample from the naive group is shown. (C) Absolute cell counts of identified myeloid cell subsets were obtained and compared. Numbers per mouse were plotted for each cell type, with data collected over 2 independent experiments and each data point representing an individual animal. Groups were compared using 2-tailed Student's $t$ test, and naive and experienced mice did not significantly differ for any cell type. AM, alveolar macrophages; CDC, conventional dendritic cells; eos, eosinophils; IM, interstitial macrophages; monos, monocytes; neutro, neutrophils; pDC, plasmacytoid dendritic cells.

reveal differences that were unappreciated from such manual analyses. We applied nearest-neighbor-based PhenoGraph clustering (33) to identify all distinct populations based on 9 parameters (FSC/SSC/CD11c/ CD45/CD64/Ly6C/Ly6G/MHC II/SiglecF) in the lungs of naive and experienced mice. CD45 cells were separated into 24 distinct clusters using this tool (Figure 3A). Three of these clusters (clusters 2, 6, and 20) differed significantly between experienced and naive lungs (Figure 3A). To visualize the complete phenotypic space of these lung immune cells, the optimized t-distributed stochastic neighbor embedding (opt-SNE) algorithm (34) was used to present multidimensional single-cell data on a single 2-dimensional plot for each of the PhenoGraph clusters (with the 20 most abundant shown in Figure 3B) or the manually gated populations (with the 7 cell assignments used in Figure 2 shown in Figure 3C). Alveolar macrophages comprised 3 clusters (clusters 2, 6, and 12), the larger 2 of which differed between groups (with cluster 2 increasing and cluster 6 diminishing in experienced lungs). Neutrophils, monocytes, eosinophils, and pDCs each fell within their own distinct clusters $(5,11,14$, and 17 , respectively), none of which changed because of experience. IMs and cDCs together fell mostly within cluster 15, but this PhenoGraph-defined cluster was further subdivided in opt-SNE maps; multiple distinct subsets of IMs were discernible, consistent with prior observations $(35,36)$, as well as multiple subsets of $\mathrm{cDCs}(37)$ that could be distinguished from those IMs. None of these IM or CDC populations appreciably differed because of experience, although changes independent of the markers used here may remain undetected. Unidentified cells using these surface markers likely included diverse lymphocytes from blood and lung tissue.

The quantitative generation and statistical comparisons of clusters (Figure 3A) combined with the opt-SNE maps of those clusters (Figure 3B) and of manually gated cell types of interest (Figure 3C) clearly revealed a major shift in alveolar macrophage phenotypes in the lungs of experienced mice, but 
A

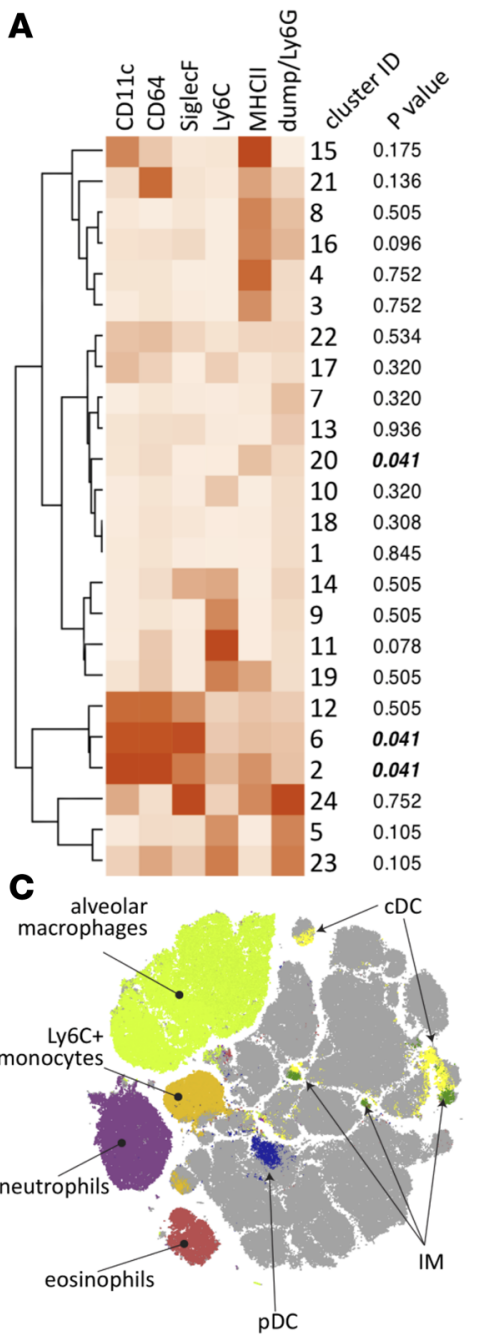

B

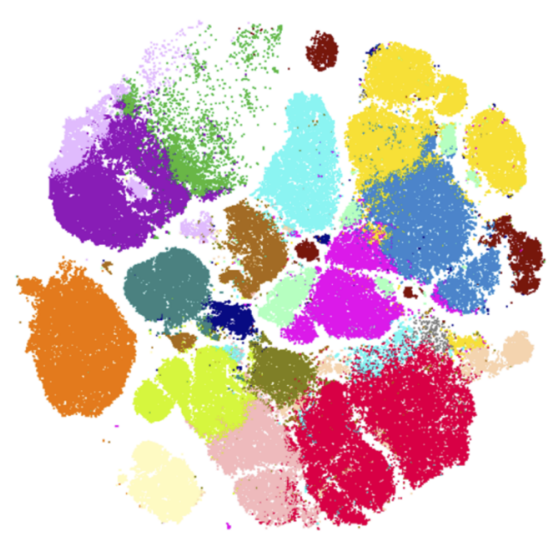

D

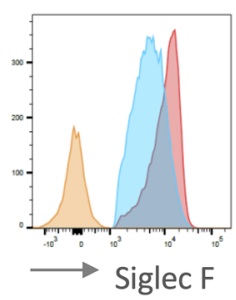

E

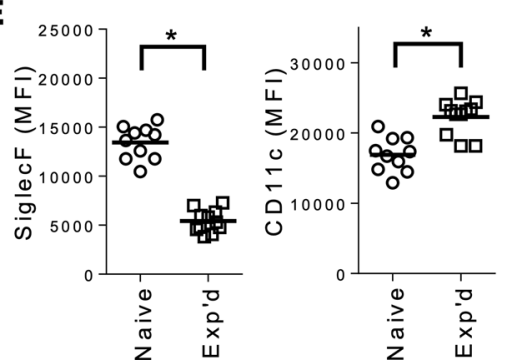

Naive mice

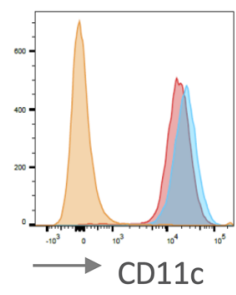

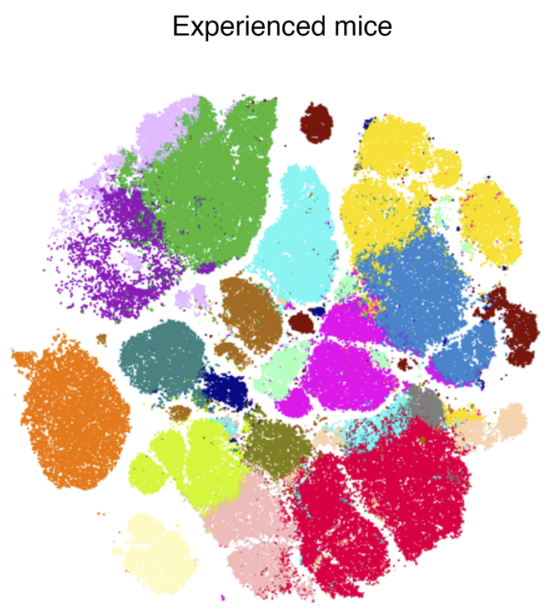

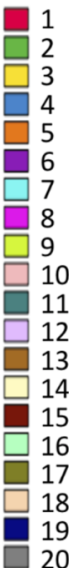

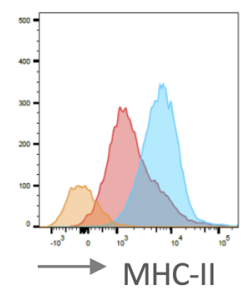

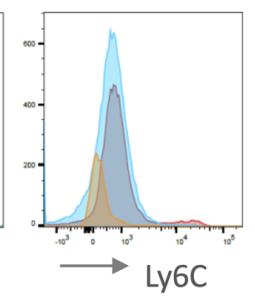

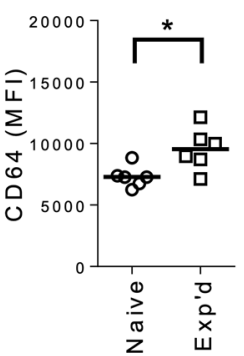
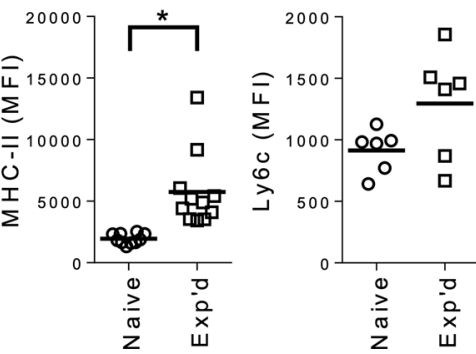

Figure 3. Myeloid cell subsets differentiating experienced mice from naive mice. For $\mathbf{A}-\mathbf{C}$, a flow cytometry data set containing equal numbers of live single-cell $C D 45^{+}$events from left lung lobes of $n=6$ mice in each of the 2 groups (naive or experienced) was phenotyped for relative surface expression of myeloid cell markers CD11c, CD64, Ly6C, Ly6G, MHC II, and SiglecF. (A) Leukocyte subsets in the lungs of naive and experienced mice. Unsupervised clustering of 24 cell subsets (numbered according to cell quantity) was generated using the PhenoGraph algorithm, with heatmap color intensity representing median surface expression for each marker across the entire data set including all cells from all mice. The Wilcoxon rank-sum test with Benjamini-Hochberg multiple-comparisons adjustments was used to compare relative numbers of events (cells) within each cluster between naive and experienced mice, with the FDR-adjusted $P$ value shown for each cluster. (B) Distributions of leukocyte phenotypes in the lungs of naive and experienced mice. The 20 most abundant PhenoGraph clusters based on multidimensional single-cell data (A) were color-coded and plotted on a 2-dimensional (2D) graph using the opt-SNE algorithm for naive and experienced mice. Opt-SNE 2D coordinates are shown on $x$ and $y$ axes. (C) Distributions of manually gated myeloid cells on the opt-SNE map. Myeloid cell-types were binned as in Figure 2 based on expression levels of the surface markers of interest. (D) Expression of surface markers across alveolar macrophages from the lungs of naive or experienced mice, depicted as a representative histogram from a single naive (red) or experienced (blue) mouse. Orange histogram represents matched fluorescence minus one negative control. (E) Comparisons of individual surface markers on alveolar macrophages between naive and experienced mice. MFI values per mouse were plotted for each surface marker, with data collected over 2-3 independent experiments and each data point representing an individual animal and horizontal lines representing group means. Asterisks $(*)$ indicate comparisons reaching statistical significance $(P<0.05)$ using unpaired 2-tailed Student's $t$ tests.

not in other recognized myeloid subsets. The majority of alveolar macrophages were in cluster 6 in naive mice, whereas most were in cluster 2 in experienced mice (Figure 3, B and C). Most prominently, experienced alveolar macrophages (gated as in Figure 2B) expressed less SiglecF and more MHC II than naive cells, across all mice examined (Figure 3, D and E). CD64 and CD11c also showed modest increases in surface expression due to experience with prior infections, despite overlap between groups (Figure 3, D and E). Ly6c (Figure 3, D and E) did not demonstrate significant differences in surface expression due to prior experience. Thus, the surface phenotype of alveolar macrophages significantly differed in mice with a prior history of resolved pneumococcal infections. This could result from long-lived alveolar macrophages that change their phenotype or from cell replacement by new alveolar macrophages with 
A 3 months since last infection
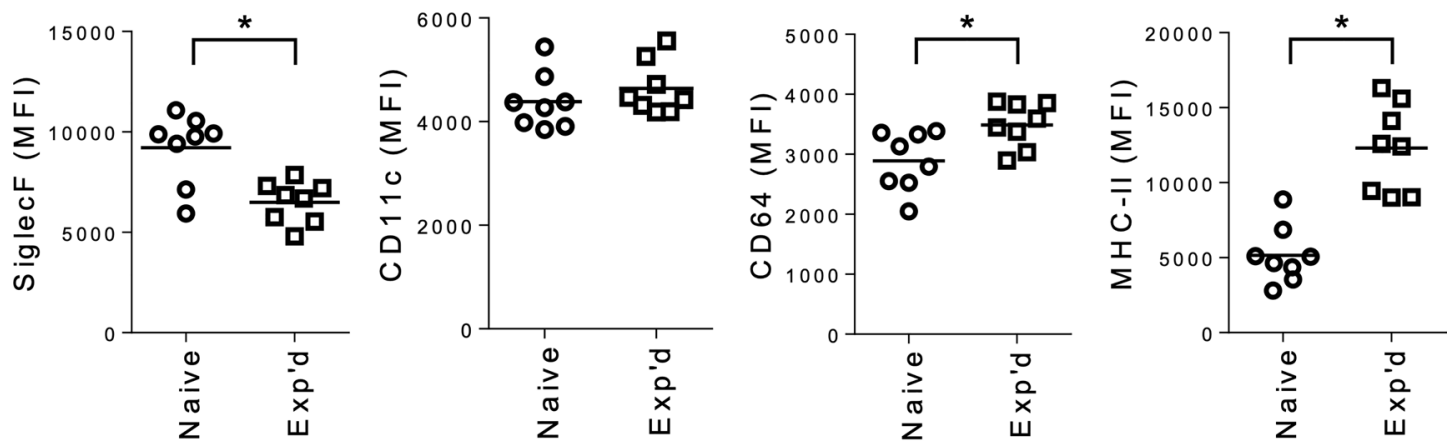

B 6 months since last infection
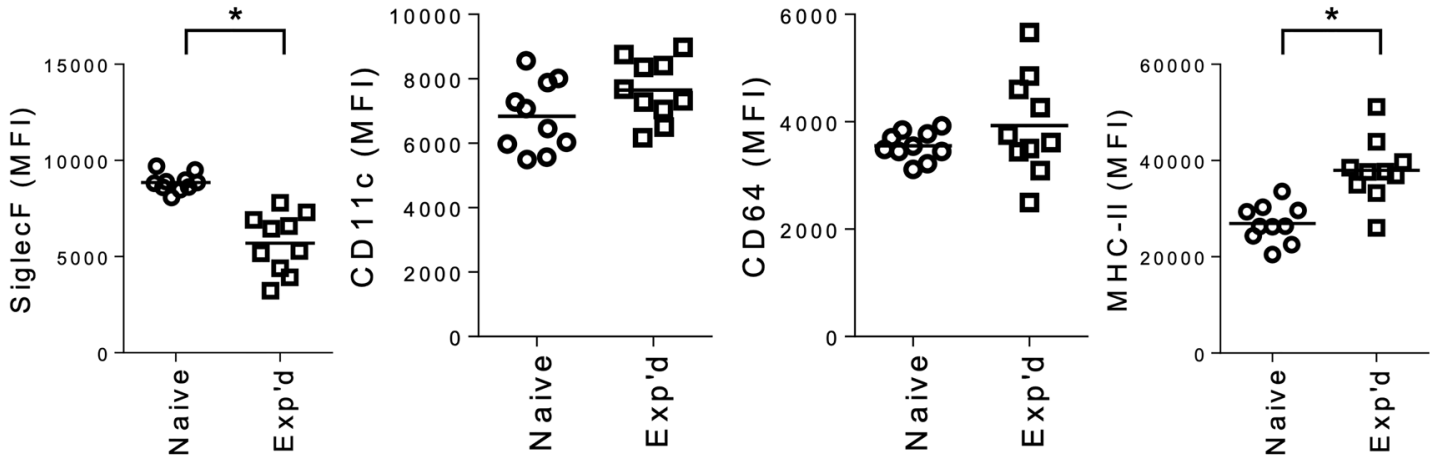

C No experimental infections (naive mice)
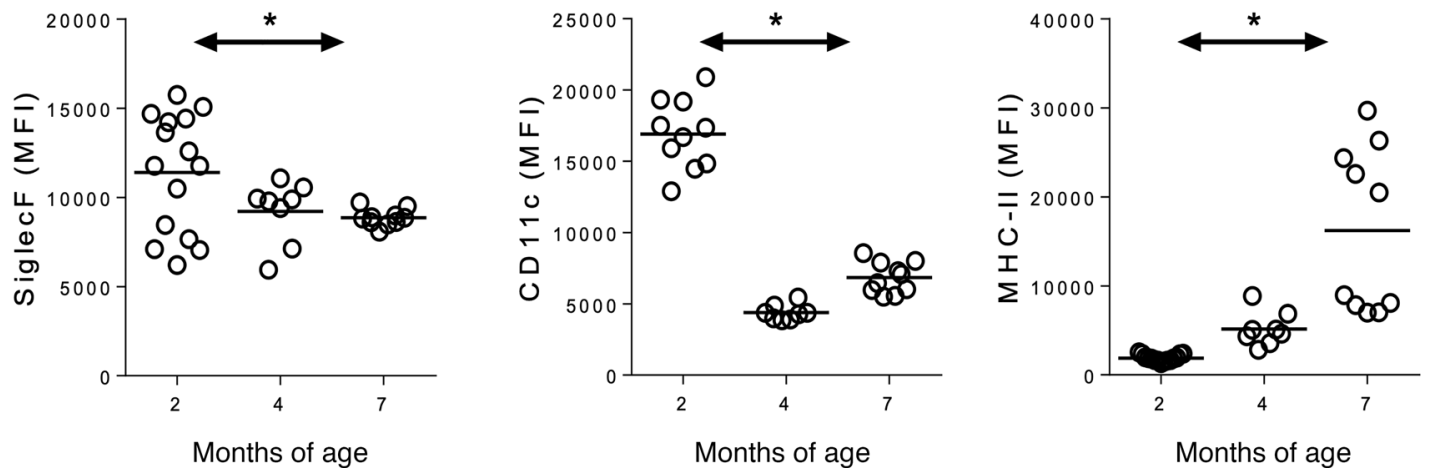

Figure 4. Effects of time on the remodeling of alveolar macrophage surface markers. Alveolar macrophages from lung single-cell suspensions of naive and experienced mice were gated as described in Figure 2, and MFI of surface markers was shown for lungs collected (A) 3 months or (B) 6 months after i.t. instillations of serotype $19 \mathrm{~F}$ pneumococcus (for the experienced mice) or sterile saline (for naive). Data in $\mathbf{A}$ and in B were each collected across 2 independent experiments, and asterisks $\left(^{*}\right)$ represent comparisons reaching statistical significance $(P<0.05)$ using unpaired 2-tailed Student's $t$ tests. (C) To determine effects of age in the absence of experimental infection, alveolar macrophages from lung single-cell suspensions were collected from naive mice of varying ages, as indicated. Data from $\mathbf{C}$ were from a single experiment, and asterisk $\left(^{*}\right)$ represents statistically significant $(P<0.05)$ effects of age using 1-way ANOVA. For all panels, each individual dot represents a single mouse, with horizontal lines representing group means.

different phenotypes. The remodeled phenotype of alveolar macrophages included decreased surface expression of SiglecF coupled with increased surface expression of MHC II.

The altered alveolar macrophage phenotype is long-lasting. To address whether the bacteria-induced remodeling of alveolar macrophages persisted over longer time frames, mice were maintained for extended periods after recovering from the initial mild and self-limiting respiratory infections. Alveolar macrophage remodeling was still observed in experienced mice compared with naive controls both 3 months (Figure 4A) and 6 months (Figure 4B) after prior infections. The decreased SiglecF and increased MHC II surface expression 
levels were preserved in the lungs of experienced mice throughout the extended time course of this experiment (Figure 4, A and B). In contrast, the modest difference between experienced and naive mice in CD11c and CD64 surface expression at 1 month after infection (Figure 3, D and E) waned at these later time points (Figure 4, A and B), suggesting that this aspect of the phenotype may persist for over a month but be less stable compared with the other surface marker changes.

The alveolar macrophage phenotype spontaneously changes over time. The experiments communicated in Figure 3 and Figure 4, A and B, were designed to determine whether the alveolar macrophages of experienced mice that had recovered from pneumococcal pneumonia differed from those of naive mice at multiple time points after prior respiratory infection. At each of the time points examined, a substantial effect of the prior infections was observed compared with age-matched mice who had previously received sterile saline vehicle instead of serotype 19F pneumococci. However, even in the absence of experimental infections, mice are exposed to microbiota in the respiratory tract (38), leading us to consider whether the phenotypes triggered by resolution of pneumococcal pneumonia might emerge spontaneously over time. To test this, we examined whether alveolar macrophages altered their surface marker phenotypes over time in naive mice. We collected lungs from naive mice of 3 ages (2, 4, or 7 months), all of which had received saline instillations at 1 month of age, and we directly compared surface marker expression by alveolar macrophages across these age groups. Interestingly, alveolar macrophage surface markers changed over time, albeit to a lesser extent and not identically to what was observed in experienced mice. There was a decrease in SiglecF and in CD11c and an increase in MHC II (Figure 4C). In conjunction with the data from Figure 3, and Figure 4, A and B, the SiglecF decrease and MHC II increase appear to be very consistent changes that are long-lasting when caused by pneumonia resolution and appearing spontaneously in mice housed in specific pathogen-free (SPF) conditions. In contrast, the CD11c change was more labile, as this marker was increased only transiently after pneumonia recovery and decreased in expression over time as mice aged in SPF housing. Altogether, these data suggest that the SiglecF and MHC II markers are characteristic of the alveolar macrophage remodeling that results from pneumonia recovery, and perhaps similarly from routine alveolar macrophage interactions with lung microbiota.

The remodeling of alveolar macrophages localizes regionally. Pneumococcal pneumonia most often presents as a lobar infection, and the resident memory lymphocytes that result after recovery from such infections are restricted to the relevant lung lobe (11). Whether and which other aspects of immune remodeling after lobar pneumonia are also restricted is unknown. Only in the infected lobe can alveolar macrophages interact directly with pneumococcus, recruited leukocytes, and local immune signals. However, systemic signals could affect macrophages in contralateral lobes, and macrophages are motile cells that can relocate over time. To assess a potential regionalization of the alveolar macrophage remodeling, we generated mice selectively infected by serotype $19 \mathrm{~F}$ pneumococci in the left lung through tracheostomy and left bronchus instillation. Left lung infections were repeated a week later, after which mice rested for at least 4 weeks in order to generate a cohort of left lobe-experienced mice (Figure 5A). Single-cell suspensions were generated separately for the right lobe and left lobe from each mouse. Compared with those in the right lobe, the alveolar macrophages from the left lobe showed decreased SiglecF, increased MHC II, and increased CD64, but no change in CD11c (Figure 5A). Thus, the majority of the remodeled alveolar macrophage phenotypes were seen to localize exclusively to cells from the tissue sites that were previously infected.

Both naive and remodeled alveolar macrophages have essential roles in defense. We tested whether the left lobe containing experienced alveolar macrophages had superior protection against diffusely distributed pneumococcus compared with the right lobes containing nonremodeled alveolar macrophages. Mice were infected with a serotype 3 pneumococcus, which is not recognized by the serotype-specific anticapsular polysaccharide antibodies that may have been generated by the prior serotype $19 \mathrm{~F}$ infections. Diffuse infections of the entire respiratory tract were accomplished by delivering serotype 3 pneumococcus intranasally, both for left lobe-experienced mice and for naive controls, which had instead previously received left lobe instillations of saline (Figure 5B). Numbers of living bacteria in each lobe were separately assessed and compared. In naive mice, the bacterial count was similar in the left and right lungs 24 hours after intranasal serotype 3 infection (Figure 5B). Experienced mice had lower bacterial burdens in both sides than did naive mice (Figure $5 \mathrm{~B}$ ), reflecting improved defense against this respiratory tract-wide infection due to prior heterotypic infections. In contrast to the naive mice, bacterial colony-forming units were diminished significantly in the left lung compared with the right in left lobe-experienced mice (Figure 5B), revealing that enhanced immune protection localizes in conjunction with alveolar macrophage remodeling. Together, the above data indicate 

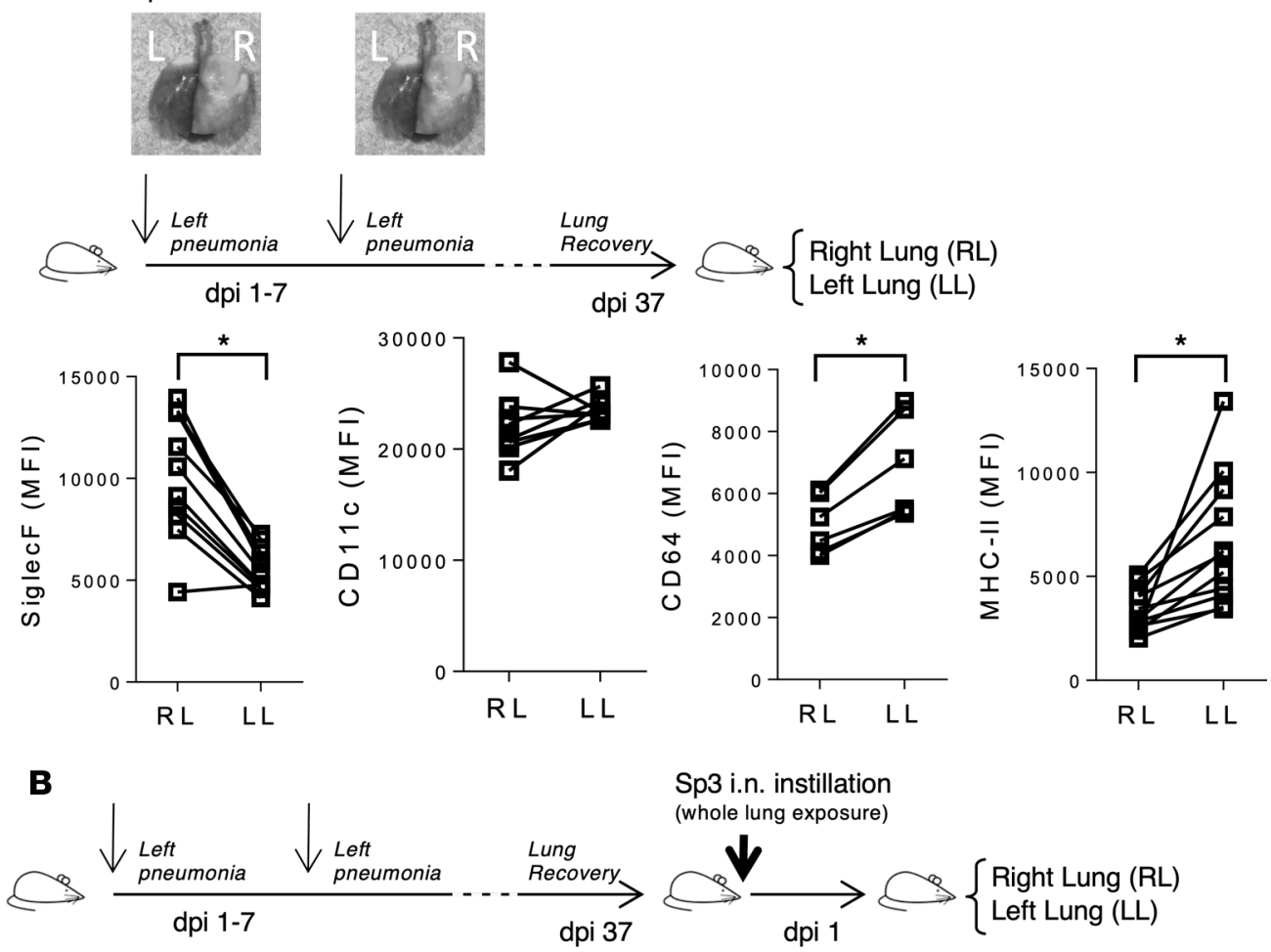

Sp3 i.n. instillation (whole lung exposure)
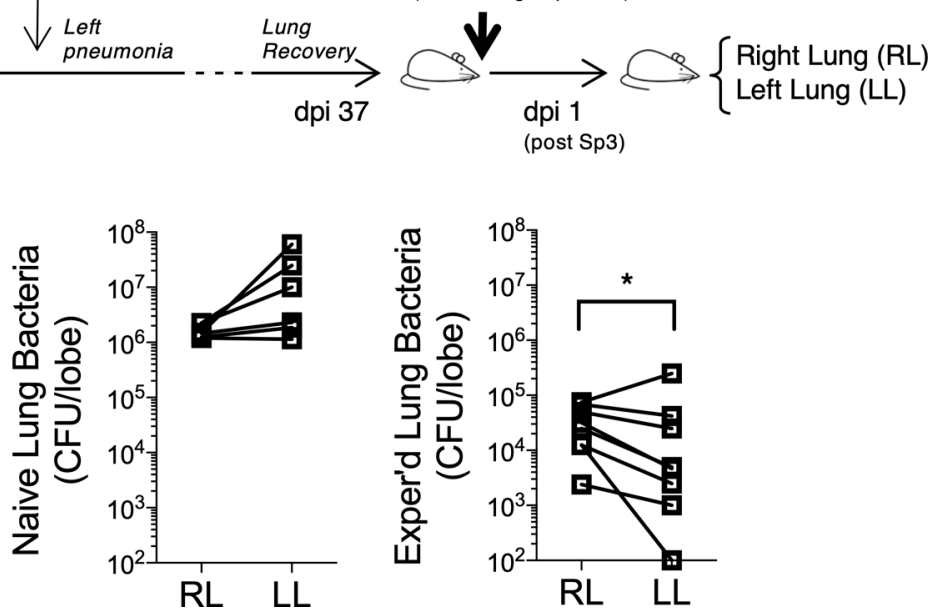

Figure 5. Regional localization of alveolar macrophage remodeling. (A) Mice were infected with 2 doses of $1 \times 10^{6}$ to $3 \times 10^{6} \mathrm{CFU}$ of serotype $19 \mathrm{~F}$ pneumococcus, with 1-week intervals between infections, and then rested at least 1 month. To generate the initial selective left lung infections, an angiocatheter was placed into the left bronchus through a tracheostomy to administer bacteria or sterile saline. Right and left lungs were harvested separately to generate single-cell suspensions. Surface marker MFI on alveolar macrophages (gated as in Figure 2) from right lobe (RL) and left lobe (LL) were compared within each mouse. Each pair of connected dots represents a single mouse. (B) A month after serotype $19 \mathrm{~F}$ pneumococcus infections of the left lung lobe, mice were challenged i.n. with $7.5 \times 10^{5}$ CFU serotype 3 pneumococcus. Bacterial burden was assessed after 24 hours in the RL versus LL. Each pair of connected dots represents a single mouse. Data were from 2 (A) or 3 (B) independent experiments. Asterisks $\left(^{*}\right)$ indicate comparisons that reached statistical significance $(P<0.05)$ using the nonparametric 2 -tailed sign test.

that the alveolar macrophage remodeling that is restricted to the previously infected lobe associates with improved antibacterial defense in that specific region during a diffuse respiratory tract infection.

To more specifically examine alveolar macrophage roles in the defense of naive and experienced lungs, we examined the effects of alveolar macrophage depletion on bacterial infections in these different settings. We sought to determine the contributions of alveolar macrophages to the defense of naive and experienced lungs by depleting them prior to serotype 3 infection. Alveolar macrophages were mostly but not completely depleted by liposomal clodronate, whether alveolar macrophages were enumerated based on morphological assessments of cells in BAL samples (Figure 6A) or by surface markers on cells in single-cell suspensions from digested lungs (Figure 6B). Similar effects of clodronate on alveolar macrophage depletion were 

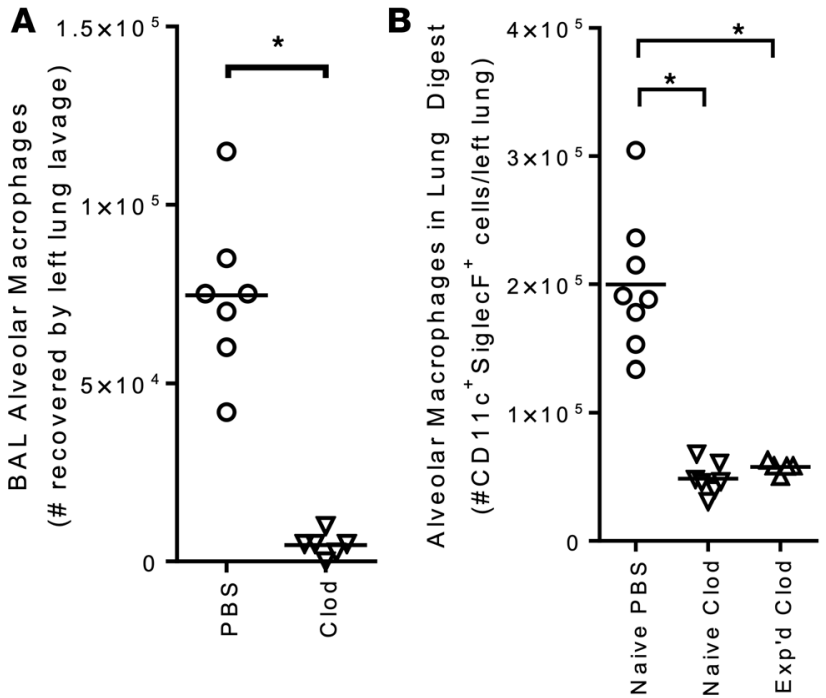

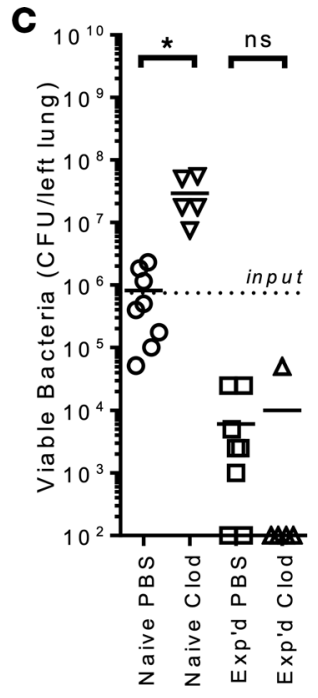

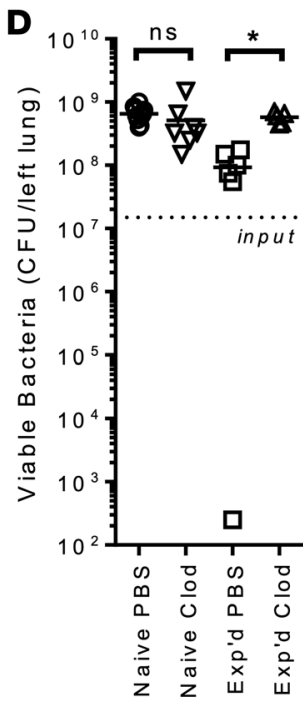

Figure 6. Alveolar macrophage roles in defending naive and experienced lungs. (A and B) Liposomal clodronate effectively depleted mouse lungs of alveolar macrophages. Mice received i.n. liposomes that contained either PBS or clodronate (Clod). (A) Alveolar macrophage numbers were assessed using microscopy and cellular morphology with BAL samples from naive mice. Every individual symbol represents a single mouse, with horizontal lines showing the mean for the group. Asterisk $\left(^{*}\right)$ represents a significant $(P<0.05)$ difference using unpaired 2-tailed Student's $t$ test. Results include data pooled from 2 independent experiments. (B) Alveolar macrophage numbers were assessed using flow cytometry and surface marker characteristics with lung single-cell suspensions from naive or experienced mice. Every individual symbol represents a single mouse, with horizontal lines showing the mean for the group. Asterisks $\left.{ }^{*}\right)$ represent significant $(P<0.05)$ differences from the naive PBS group using 1-way ANOVA and Holm-Šídák post hoc multiple-comparisons tests. Results include data from 2 independent experiments. (C and D) Naive or experienced mice received i.n. liposomes that contained either PBS or clodronate 72 hours prior to i.n. infections with either (C) a medium dose $\left(0.75 \times 10^{6} \mathrm{CFU}\right)$ or (D) a high dose $\left(15 \times 10^{6} \mathrm{CFU}\right)$ of serotype 3 pneumococcus. Infecting dose is depicted on graphs with a dotted line. Lung bacterial burdens were determined 24 hours after infection. Every individual symbol represents a single mouse, with horizontal lines showing mean for the group. Asterisks $\left(^{*}\right)$ indicate significant $(P<0.05)$ effects of clodronate using 2 -way ANOVA on log-transformed data and Holm-Šídák post hoc multiple-comparisons tests (ns, not significant). Results include data from 3 independent experiments for each bacterial dose.

observed whether mice were naive or experienced (Figure 6B). We infected the liposome-pretreated mice with $0.75 \times 10^{6} \mathrm{CFU}$ of serotype 3 pneumococcus. In naive mice, the bacteria isolated from lungs after 24 hours approximately equaled what was initially delivered, but depletion of alveolar macrophages significantly increased bacterial burdens (Figure 6C). Thus, alveolar macrophages are essential to immunity in naive mice. Experienced mice had substantially better protection against pneumococcus than did naive mice, eliminating nearly $99 \%$ of living bacteria in less than 24 hours (Figure 6C). This highly effective protection in experienced lungs was unimpeded by alveolar macrophage depletion (Figure 6C). Thus, in contrast to naive lungs, the ready elimination of pneumococcus in the setting of heterotypic immunity did not require a full set of alveolar macrophages, likely because of other elements of immune protection gained after prior experience, such as lung CD4+ Trm cells accelerating neutrophil recruitment $(11,39)$. To further challenge the lung immune system, we infected mice with higher doses of bacteria, $15 \times 10^{6} \mathrm{CFU}$. Under such conditions, the naive mice were unable to control bacterial growth, and depletion of alveolar macrophages had no effect (Figure 6D). Bacteria grew over 24 hours in the lungs of experienced mice as well, but this growth was significantly constrained, as demonstrated by lower lung colony-forming units in the experienced lungs compared with the naive lungs (Figure 6D). This immune control in experienced lungs was entirely dependent on alveolar macrophages, because alveolar macrophage depletion significantly increased bacterial burdens and rendered them akin to what was observed in naive lungs (Figure 6D). Thus, in the setting of severe infection, the remodeled alveolar macrophages are essential for the improved defense of lungs with naturally acquired heterotypic immunity.

Alveolar macrophages in experienced lungs have altered metabolism. Long-term alveolar macrophage adaptations due to prior interactions with microbial stimuli are suggestive of trained immunity, and trained immunity of monocytes and macrophages is girded by metabolic reprogramming (22). Therefore, we tested whether metabolomes of alveolar macrophages differed in experienced lungs compared with naive lungs, using liquid chromatography-mass spectometry-based (LC/MS-based) metabolic profiling to assess metabolites. We compared metabolomes of BAL cells from naive mice with those from experienced mice that recovered from diffuse bilateral pneumonias. From the LC/MS spectra, 73 metabolites were identified and 
- Naive mice

A $\quad$ Experienced mice

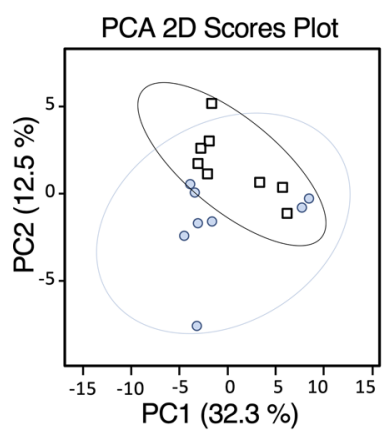

B

Left-experienced mice

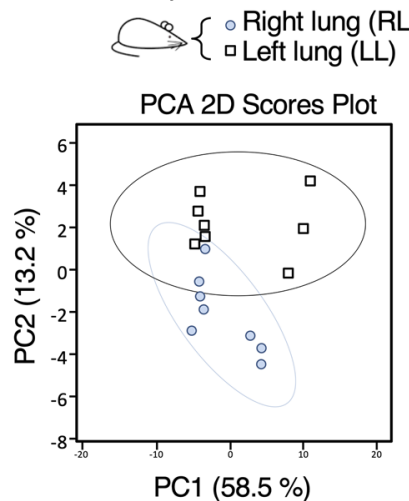

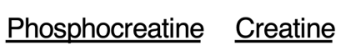

FDR- $\mathrm{p}=0.03 \quad$ FDR- $\mathrm{p}=0.01$ Fold $=4.4 \mathrm{x} \quad$ Fold $=3.1 \mathrm{x}$

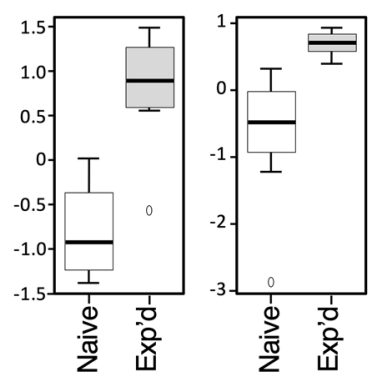

C

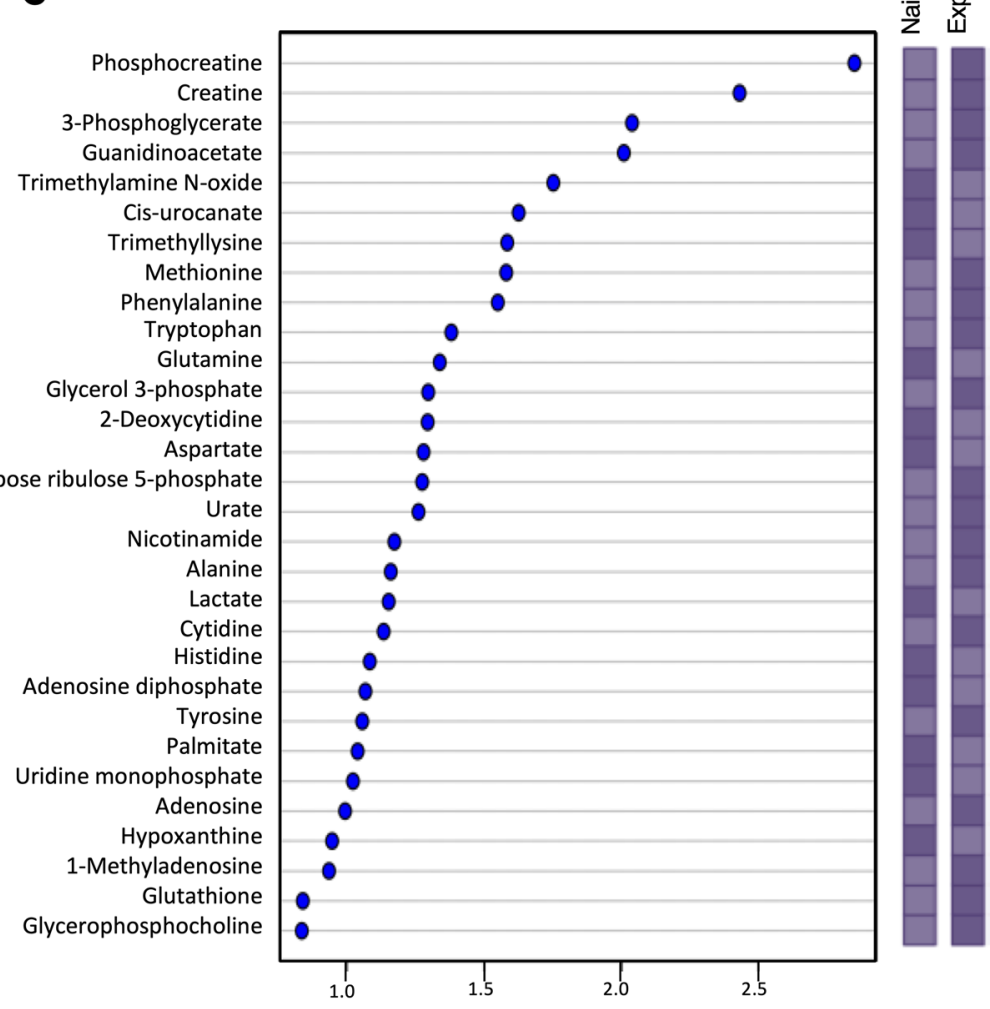

Figure 7. Altered metabolomes of alveolar macrophages after resolution of pneumococcal infections. (A) Score plot of PCA performed on the LC/MS data of alveolar macrophages lavaged from whole lungs of naive (circle) and experienced (square) mice. The explained variances are shown in parentheses. (B) Score plot of PCA performed on the LC/MS data of alveolar macrophages lavaged selectively from the right (circle) or left (square) lung lobes of left-experienced mice that recovered from prior lobar pneumonias in the left lobe. The explained variances are shown in parentheses. For $\mathbf{A}$ and $\mathbf{B}$, features selected by volcano plot that reached the threshold of 2 times fold change and $P<0.01$ by 2-tailed Student's $t$ tests were represented in box plots, log-transformed and normalized to a constant sum. (C) Metabolites identified by PLS-DA and VIP scores (comparison of metabolomes of alveolar macrophages from naive and experienced mice). Significant contribution was considered if VIP $>1$. Colored boxes on the right indicate relative concentrations of the corresponding metabolite (elevated in condition with darker box). Two independent experiments with 4 mice per group were performed $(n=8)$ for both designs.

compared between the experimental groups using an unsupervised multivariate analysis based on principal component analysis (PCA). PCA differentiated experienced from naive alveolar macrophages based on their metabolomes (Figure 7A), with the first principal component alone accounting for $32 \%$ of the variance. Examination of the individual metabolites that changed between naive and experienced mice revealed that phosphocreatine (fold change $=4.4$, and FDR-adjusted $P=0.03$ ) and creatine (fold change $=3.1$, and FDR-adjusted $P=0.01$ ) differed prominently (Figure 7A). Guanidinoacetate also showed statistically significant differences between alveolar macrophages from experienced and naive lungs (Supplemental Figure 1; supplemental material available online with this article; https://doi.org/10.1172/jci.insight.133042DS1). Thus, 3 of the 5 metabolites that reached significance were immediately adjacent to each other within a distinct metabolic pathway (Supplemental Figure 2).

Because the remodeling of alveolar macrophages localizes regionally, we leveraged lobar pneumonias to assess whether metabolic changes in alveolar macrophages differed throughout lung regions. We compared whole-cell metabolomes of cells lavaged from right (uninvolved) and left (previously infected) lobes from mice that recovered from lobar rather than diffuse pneumonias. PCA clearly discriminated the metabolomes of alveolar macrophages collected from lung lobes with different infection histories (Figure 7B), with the first principal component alone accounting for $58 \%$ of the variance. As in studies of naive versus experienced mice, increased phosphocreatine was the most dominant signal (Figure $7 \mathrm{~B}$ ), increased in cells lavaged from the left lung compared with the right (fold change $=2.8$, and FDR-adjusted $P=0.01$ ). Creatine showed a shift in that direction that did not reach significance (fold change $=1.3$, and FDR-adjusted $P=0.07)$. However, the fact that right and left metabolomes differed and that 


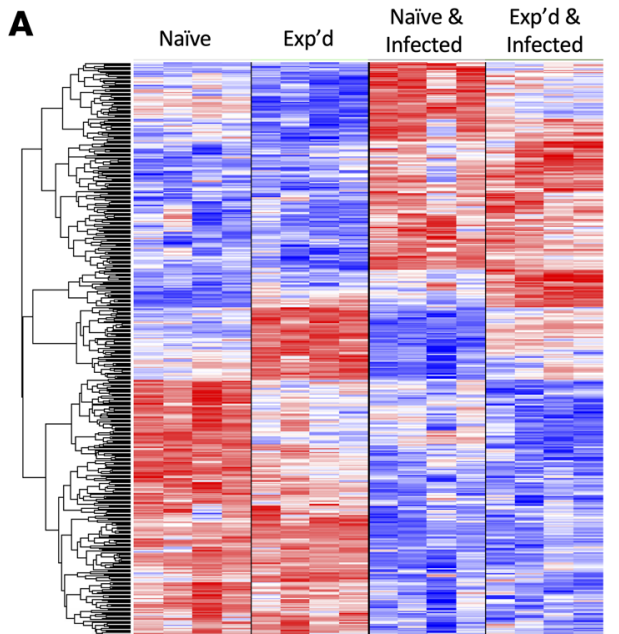

B

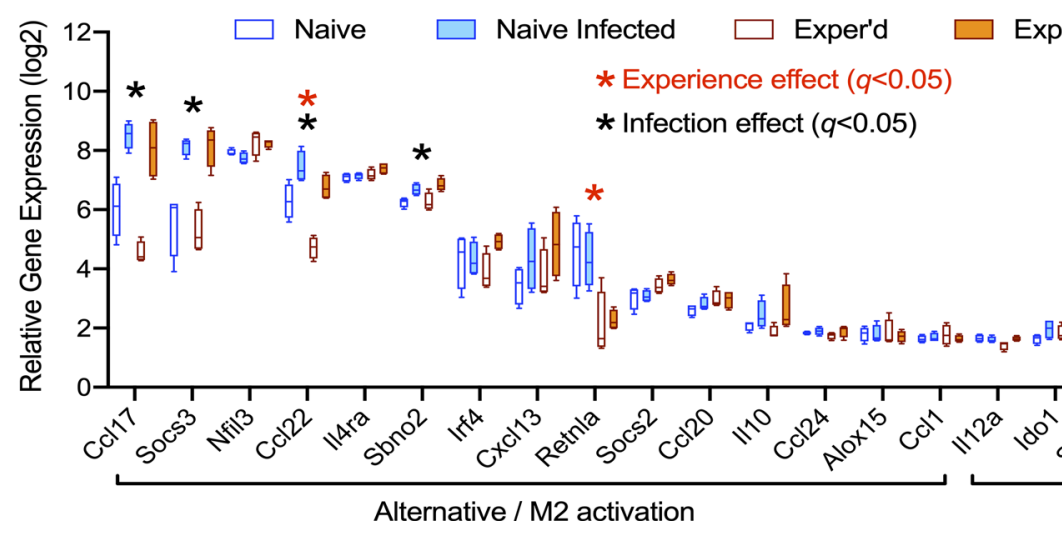

C

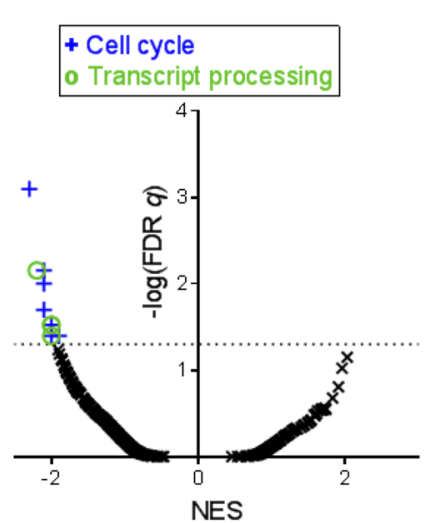

Pathways changed at baseline Pathways changed during infection

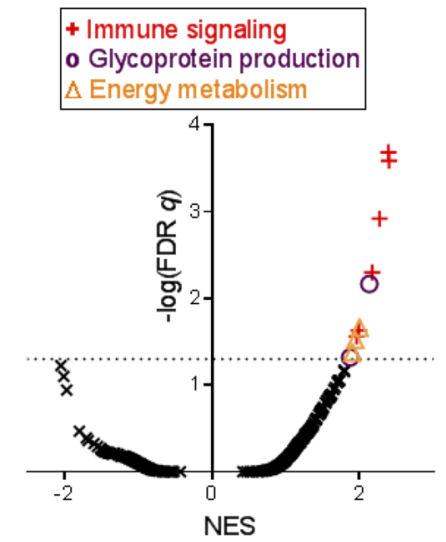

NES

Exper'd Infected $\quad * \quad *$

* $\stackrel{*}{*} \stackrel{*}{*}$

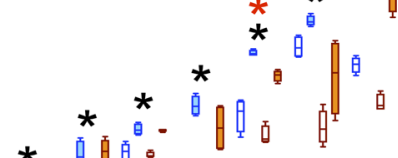

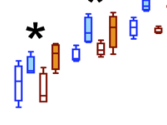

$\coprod_{0}$

Classical / M1 activation

Figure 8. Transcriptome remodeling of experienced alveolar macrophages, at rest and during infection. (A) Hierarchical clustering and heatmap of 443 transcripts differentially expressed with FDR $q<0.05$ in alveolar macrophages of experienced and naive mice. Each column represents a mouse and each row a gene. Color-coding indicates directionality (with blue, white, and red showing below-, at-, or above-average expression, respectively). (B) Relative expression of genes associated with "alternative/M2" or "classical/M1" activation states in mouse macrophages (42). Box, line, and whisker for each mRNA represent quartiles, median, and range, respectively, for $n=4$ mice per group in the genome-wide transcriptome analyses. (C and $\mathbf{D}$ ) Plots of reactome pathways resulting from GSEA analyses of the transcriptome profiles of alveolar macrophages from experienced compared with naive lungs, at rest (C) or 4 hours after infection with serotype 3 pneumococcus (D). All pathways that crossed the FDR $q<0.05$ threshold were examined (listed in Tables 1 and 2), colored differently from black in the figures, and grouped by manual inspection (as detailed in Tables 1 and 2) into overarching pathways that were communicated in the figures via inset boxes. Normalized enrichment scores (NESs) below 0 indicate pathways decreased in the experienced lungs, while positive NESs indicate pathways increased in experienced lungs.

phosphocreatine was a discriminating factor supports the idea that changes to creatine metabolism may localize to alveolar macrophages in the region that previously experienced pneumococcal infection.

To further determine the metabolites that drive the differences between the groups, we used supervised multivariate analysis based on partial least squares-discriminant analysis (PLS-DA) to compare metabolomes of alveolar macrophages from naive and experienced mice. We observed a correct clustering by classification (data not shown) with accuracy, $R^{2}$, and $Q^{2}$ equal to $0.81,0.89$, and 0.54 , respectively. On the basis of the PLS-DA model, metabolites that contributed significantly to the classification were highlighted and ranked by the variable importance in projection (VIP) values that estimate the importance of each variable in the projection (Figure 7C). Phosphocreatine was the most discriminant result (VIP > 2.5), with creatine and guanidinoacetate prominent as well. Altogether, these data reveal that alveolar macrophages of experienced lungs have altered metabolic activities, with elevated phosphocreatine and its precursors being cardinal features.

Transcriptomes of remodeled mouse alveolar macrophages differ at baseline and in response to pneumococcus, better resembling human alveolar macrophage responses. To define the transcriptomes of alveolar macrophages sorted from lung digests of naive or experienced mice, we used genome-wide expression profiling. We used FACS to isolate alveolar macrophages from both groups of mice at baseline, to determine whether these 
Table 1. Pathways downregulated at baseline in transcriptomes from experienced compared with naive alveolar macrophages

Down in experienced lungs

Up in experienced lungs

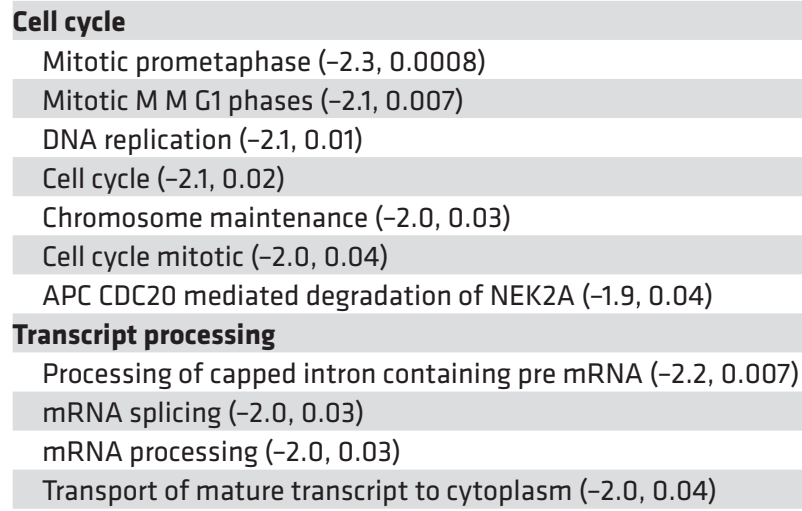

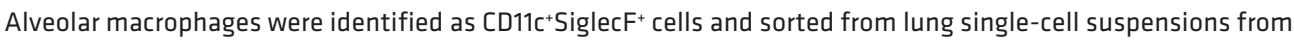
experienced or naive mice, at baseline (no ongoing infection). All reactome pathways that differed by FDR $q<$ 0.05 are included in the table, grouped by manual inspection. NES and $q$ values for each pathway are indicated in parentheses (NES, q).

cells were transcriptionally altered at rest. Pneumococcal infection dramatically and rapidly changes gene expression in lung cells $(40,41)$, so we also collected cells after 4 hours of serotype 3 pneumococcal pneumonia in order to determine whether alveolar macrophages in the better protected experienced lungs responded differently to pneumococcus. A total of 4158 transcripts significantly (FDR $q<0.05$ ) differed in alveolar macrophages because of pneumococcal lung infection, reflecting changes to more than a tenth of the transcriptome. On top of this, a substantial set of genes was significantly altered by prior history of resolved infections; 443 genes differed with FDR $q<0.05$ due to prior history of resolved infections (Figure $8 \mathrm{~A})$, more than a tenth the size of the total effect of acute infection. These data reveal that resolution of pneumonia results in substantial modifications to the transcriptomes of alveolar macrophages.

Macrophages have been ascribed phenotypes of "classical" or M1 activation versus "alternative" or M2 activation, based on in vitro experiments skewing cells in those directions (42). However, the alveolar macrophages from pneumococcus-infected or -experienced lungs did not display such skewing (Figure 8B). Although experience with prior pneumococcal infections altered expression of some of these genes (including decreases in Ccl22, Retnla, Nfkbiz, Marco, and Tnf), the effects of experience did not push alveolar macrophages toward either of these 2 previously defined polarized directions (Figure 8B). Using gene set enrichment analyses (GSEAs) to connect these transcriptome changes to biological pathways in a more open-ended fashion, we found that the manner in which alveolar macrophages in experienced lungs differed from alveolar macrophages in naive lungs depended dramatically on whether there was an ongoing infection. In the lungs at baseline (without an ongoing infection), no pathways were significantly (FDR $q<0.05$ ) increased, but multiple pathways were diminished in experienced compared with naive alveolar macrophages (Figure $8 \mathrm{C}$ and Table 1). The significantly altered pathways all aligned with decreased cell cycle activity or decreased processing of mRNA transcripts. This suggests that the alveolar macrophages from experienced lungs tend to be more quiescent under resting, homeostatic conditions. In contrast, during an acute infection, no pathways were significantly decreased, but multiple pathways were increased in experienced compared with naive alveolar macrophages (Figure 8D and Table 2). These pathways aligned with increases in immune signaling, glycoprotein production, and energy metabolism.

The transcripts that increased due to prior experience and differed most significantly (lowest FDR $q$ values) compared with naive lung macrophages included immune mediators as well as diverse other factors relating to metabolism and other cellular activities (Figure 9A). The changes in immune signaling differed from those observed after viral exposures $(15,23)$. Although relative expression of CXCL1 and CXCL2 is the dominant signal altered in alveolar macrophages from lungs previously infected with a virus $(15,23)$, neither of those chemokines was changed in alveolar macrophages due to prior infection with pneumococcus (Figure 9B). 
Table 2. Pathways upregulated during infection in transcriptomes from experienced compared with naive alveolar macrophages

Down in experienced lungs

None
Up in experienced lungs

Immune signaling

IFN gamma signaling $(2.4,0.0002)$

IFN alpha beta signaling $(2.4,0.0003)$

Interferon signaling $(2.3,0.001)$

Cytokine signaling in immune system $(2.2,0.005)$

Antigen presentation ${ }^{A}(2.0,0.02)$

Negative regulators of RIC I MDA5 signaling $(2.0,0.03)$

Glycoprotein production

Asparagine $\mathrm{N}$-linked glycosylation $(2.1,0.007)$

$\mathrm{N}$ glycan nascent protein ${ }^{\mathrm{B}}(1.9,0.05)$

Energy metabolism

Respiratory electron transport $(2.0,0.02)$

ATP chemiosmotic coupling $\mathrm{C}(1.9,0.03)$

Citric acid cycle TCA cycle $(1.9,0.04)$

Alveolar macrophages were identified as ${\mathrm{CD} 11 \mathrm{c}^{+} \text {SiglecF}}^{+}$cells and sorted from lung single-cell suspensions from experienced or naive mice, during pneumonia (acute infection, 4 hours) caused by i.t. instillation of serotype 3 pneumococcus. All reactome pathways that differed by FDR $q<0.05$ are included in the table, grouped by manual inspection. NES and $q$ values for each pathway are indicated in parentheses (NES, $q$ ). Some pathway names were abridged from the following. ${ }^{A}$ Antigen presentation folding assembly and peptide loading of class I MHC. ${ }^{B}$ Biosynthesis of the $\mathrm{N}$ glycan precursor dolichol lipid linked oligosaccharide LLO and transfer to a nascent protein. 'Respiratory electron transport ATP synthesis by chemiosmotic coupling and heat production by uncoupling proteins.

Both CXCL1 and CXCL2, as well as other chemokines, were increased due to acute pneumonia but unaffected by prior experience. However, several chemokines were affected by resolution of pneumococcal pneumonia, including an increase in Ccxl9 mRNA as well as decreases in Ccxl14, Ccl22, and Cx3cl1 mRNAs (Figure 9B). This suggests that how the alveolar macrophages remodel after infection depends on the type of infection (e.g., microbe, severity, location, and duration). Phagocytosis receptors were also altered by prior experience, including increased expression of Msr1 (encoding scavenger receptor A) and Clec12a (encoding dectin-1), along with decreases in Cd36 and Marco (Figure 9C). Thus, multiple immune mediators were altered at the transcript level.

Monocyte-derived alveolar macrophages after clodronate challenge exhibit a very small set of transcript changes compared with autochthonous alveolar macrophages (19). Probing the genes differentiating monocyte-derived alveolar macrophages after clodronate challenge (19) revealed a strong but not universal enrichment of this signature in the alveolar macrophages of experienced lungs (Figure 9D), supporting the interpretation that monocyte recruitment and differentiation to alveolar macrophages occurred after resolution of pneumococcal pneumonia. However, the pronounced differences observed in the pneumococcus-experienced lungs far exceeded the few differences ascribed to alveolar macrophages of monocytic origin (19). For example, Figure 9A shows many genes recognizably elevated in resting alveolar macrophages due to prior pneumococcal experience, only 1 of which (Apbb2) changes in alveolar macrophages of monocytic origin after clodronate (19). In addition, 1 signature gene (Retnla) significant in both cases trended in opposite directions in the clodronate and pneumococcus studies (Figure 9D). These observations reveal that pneumococcus influenced alveolar macrophages beyond effects on cell turnover. Recovery from pneumococcal pneumonia reprograms alveolar macrophages in ways that are distinct from recovery after influenza, adenovirus, or clodronate challenges.

To determine whether alterations implicated by transcriptome profiling influence immune functionality, we examined protein expression levels for some of the known immunomodulatory genes altered by prior infection history. Examination of 3 of the most significantly altered surface marker transcripts (Figure 9A) revealed that their protein products (CCR5, CD93, and scavenger receptor A) were all significantly changed in the same direction as their mRNAs (Figure 9E). However, there were discrepancies as well between transcript and protein changes, as should be expected. For example, the transcript for CD36 was significantly decreased (Figure 9C), but CD36 baseline protein expression was unaffected by prior experience (Figure 9E). Of the 4 surface markers measured at the protein level in Figure 3, Figure 4, and Figure 5, the transcript for SiglecF decreased 
A MOST SIGNIFICANT \& UP IN EXPERIENCED LUNGS

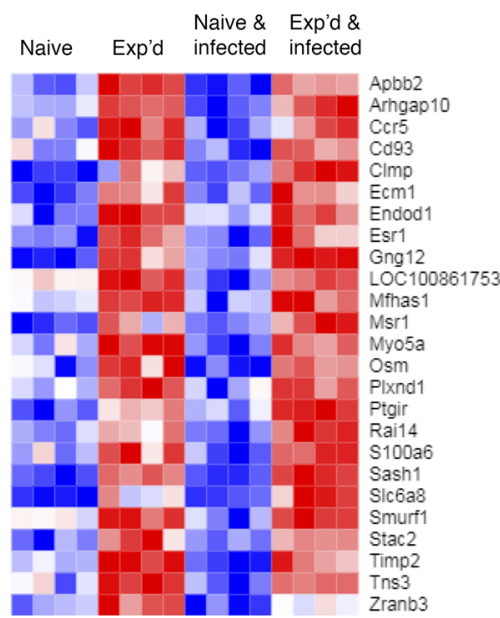

B

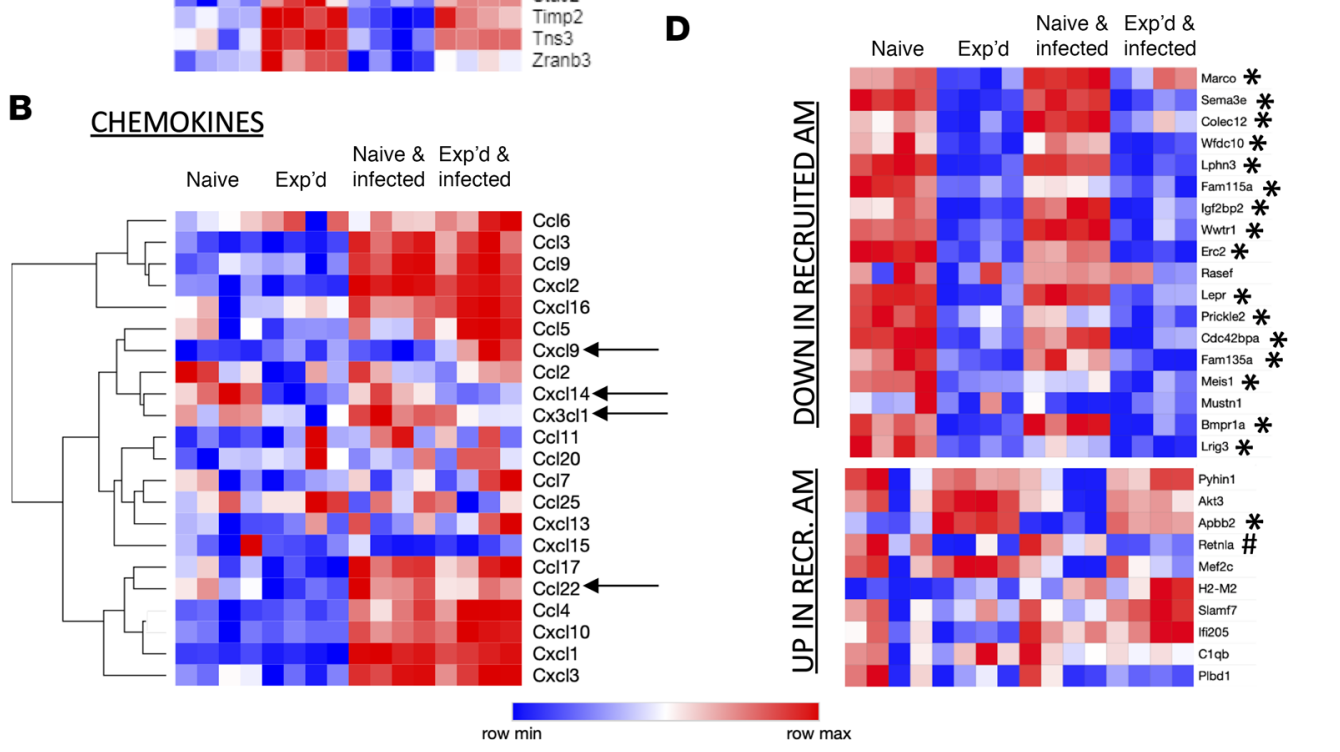

\section{PHAGOCYTOSIS RECEPTORS \\ E}

Naive \& Exp'd \& Naive Exp'd infected infected

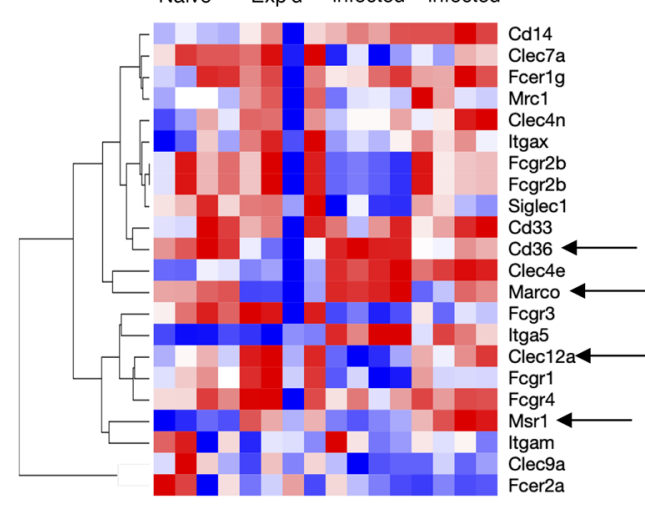

D

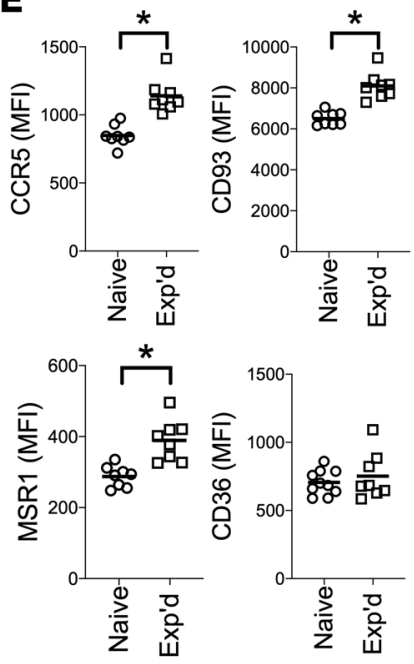

$\mathbf{F}$
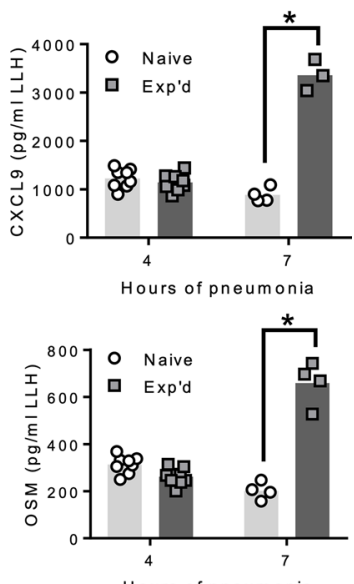

Figure 9. Transcripts and proteins altered by prior pneumonia experience. Transcripts were culled from the full data sets of alveolar macrophages from naive or experienced mice with or without acute (4-hour) infections by serotype 3 pneumococcus and presented in A-D. (A) The 25 transcripts that increased most significantly due to past history of infections. These had the lowest FDR $q$ values for effect of pneumonia history and were increased rather than decreased in alveolar macrophages of experienced lungs. (B) The chemokine transcripts detected in alveolar macrophages. Arrows indicate FDR $q<0.05$ for effect of pneumonia history. (C) The phagocytosis receptor transcripts detected in alveolar macrophages. Arrows indicate FDR $q<0.05$ for effect of pneumonia history. (D) Transcripts differentiating recruited versus autochthonous alveolar macrophages in prior studies of replacement after clodronate depletion (19). Asterisks $\left(^{*}\right)$ indicate FDR $q<0.05$ for effect of pneumonia history, with changes in the direction consistent with monocyte-derived alveolar macrophages in the experienced group, and hashtag $(\#)$ indicates FDR $q<0.05$ for effect of pneumonia history, with changes in the opposite direction. (E) Surface protein levels of surface markers implicated in transcriptome studies as differing between naive and experienced mice at the mRNA level. MFI values on alveolar macrophages were plotted for each surface marker, with data collected over 2-3 independent experiments, and each data point representing an individual animal, and horizontal lines representing group means. Asterisks $\left(^{*}\right)$ indicate comparisons reaching statistical significance $(P<0.05)$ using unpaired 2-tailed Student's $t$ tests. $(\mathbf{F})$ Protein levels of soluble cytokines implicated in transcriptome studies as differing between naive and experienced mice at the mRNA level. Concentrations were quantified by ELISA in left lung homogenates (LLH) from naive or experienced mice infected 4 or 7 hours with serotype 3 pneumococcus. Data were collected over 2-3 independent experiments, with each data point representing an individual animal and bars representing group means. Asterisks $\left(^{*}\right)$ indicate comparisons reaching statistical significance $(P<0.05)$ using 2-way ANOVA.

significantly (FDR $q=0.008$ ), whereas transcripts for CD11c, CD64, and MHC II did not change significantly. Among soluble immune mediators altered by prior experience in the macrophage transcriptomes (Figure 9 , A and B), Cxcl9 was of interest for being the only chemokine increased by experience and Osm for encoding oncostatin $\mathrm{M}$, which enhances lung neutrophil recruitment by stimulating CXCL5 expression in epithelial cells $(39,43)$. Both of these cytokines were measured by ELISA in lung homogenates at early time points of pneumonia in naive and experienced mice, and both were increased at the protein level by 7 hours of infection (Figure 9F). These results support the concept that transcriptomic changes observed in alveolar macrophages may influence the abundance of proteins with known immunomodulatory activity in the lung. 
A Transcripts (FDR $q<0.05)$

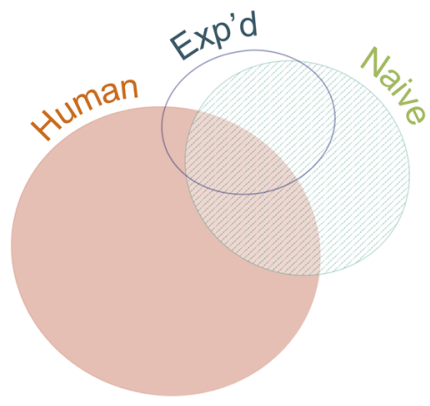

C

Human vs naive mice

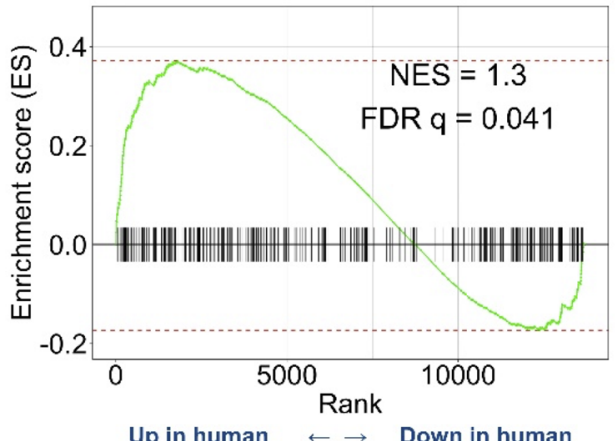

B Pathways (FDR $q<0.01)$

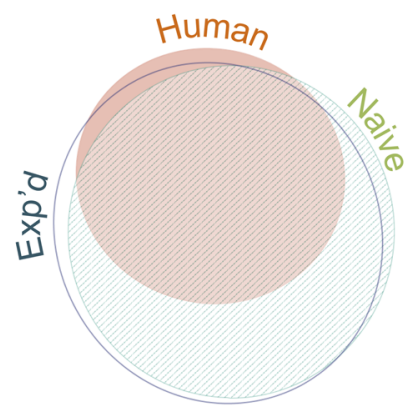

D

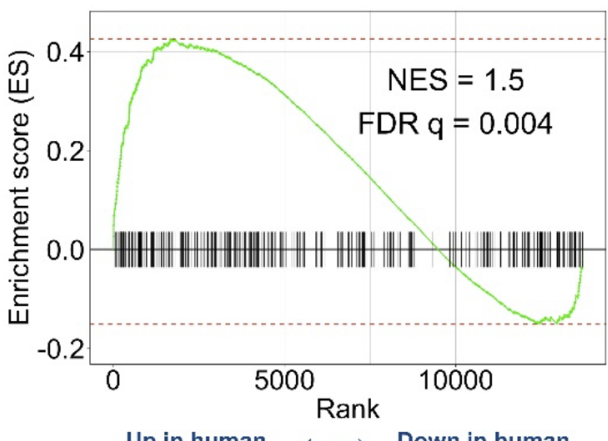

Figure 10. Comparison of mouse and human alveolar macrophage responses to pneumococcal infection. (A and B) Euler diagrams representing relative sizes and overlap of $(\mathbf{A})$ gene sets or (B) biological pathways that were significantly altered by pneumococcal stimulation of mouse alveolar macrophages that were naive or experienced (data sets collected here) or of human alveolar macrophages from Bewley et al. (40). (C and D) CSEA comparisons of human alveolar macrophage responses to pneumococcus, from Bewley et al. (40), with mouse alveolar macrophage responses to pneumococcus (from data collected here), including alveolar macrophages from mice that were (C) naive or (D) experienced.

Connecting to the metabolic changes observed in experienced alveolar macrophages (Figure 7 and Supplemental Figure 1), the transcriptomic analyses revealed that mediators of creatine metabolism (44) were altered in alveolar macrophages from experienced lungs (Supplemental Figure 2). Among the 4 creatine kinase genes, alveolar macrophages expressed $C k b$, while $C k m, C k m t 1$, and $C k m t 2$ were at most minimally expressed. There were no significant differences in $C k b$ mRNA expression due to infection history (FDR $q=0.98$ ), suggesting kinase expression is not responsible for altered phosphocreatine content. However, other genes in the creatine metabolism pathway were significantly altered (Supplemental Figure 2). The Gatm gene encoding the commitment step of the creatine biosynthesis pathway was significantly higher due to infection history. The Slc6a8 gene encoding the plasma membrane importer of creatine was one of the most significantly altered transcripts due to infection history (Figure 9A and Supplemental Figure 2). These studies implicate both the generation and import of creatine in alveolar macrophages as potential contributors to the altered metabolism of alveolar macrophages after infection resolution.

To examine whether the mouse alveolar macrophage responses to pneumococcus observed here were relevant to humans, and whether the prior lung infection history of mice influenced their relationships to human alveolar macrophages, we compared our transcriptome data sets to relevant data collected from human samples (40). The human studies relied on alveolar macrophages collected by BAL and then cultured with or without in vitro serotype 14 pneumococcal infection for 4 hours. The mouse data relied on alveolar macrophages sorted from single-cell suspensions from lungs with or without in vivo serotype 3 pneumococcal infection for 4 hours. The number of human transcripts that changed with FDR $q<0.05$ because of pneumococcus in vitro was larger than that observed in mouse cells in vivo from naive or experienced lungs (Figure 10A). Only a fraction of the transcripts that changed were identical across species and studies, about $22 \%$ of the human transcriptome responses or $38 \%$ of the mouse transcriptome responses (Figure $10 \mathrm{~A})$, highlighting differences between the in vitro human experiments and the in vivo mouse experiments. 
However, when the pathways that were associated with these transcriptome changes were compared, there was a marked convergence (Figure 10B). Thus, similar biological responses were stimulated in both the in vitro human experiments and the in vivo mouse experiments. We used GSEA to specifically determine whether the experienced mouse alveolar macrophage responses were more similar or less similar to the human responses than those of naive mouse alveolar macrophages were. Both the naive and the experienced alveolar macrophage responses in mice were significantly concordant with those of the human alveolar macrophages (Figure 10, C and D). However, the similarities to human alveolar macrophage responses were larger (NES shift to 1.5 from 1.3) and more precise (FDR $q$ shift to 0.008 from 0.035 ) for the alveolar macrophages from experienced compared with naive mouse lungs (Figure 10, C and D). These data suggest that mouse alveolar macrophages respond to pneumococcus similarly to human alveolar macrophages, and a history of respiratory infections makes mouse alveolar macrophage responses even more humanlike.

\section{Discussion}

Our results demonstrate that the pool of alveolar macrophages is persistently altered after resolution of prior bacterial pneumonias. Although the numbers of alveolar macrophages in naive and experienced lungs are similar, they differ in surface receptors, metabolomes, transcriptomes, and responses to subsequent pulmonary infections. Although prior studies of immune remodeling after infection highlight cells newly accumulating in experienced lungs (3, 8, 10-12), our study complements another (23) in emphasizing that the phenotype of cells whose numbers are similar between naive and experienced lungs may be importantly modified by prior infection history. These studies demonstrate that alveolar macrophage remodeling is a component of the immunological tone of lungs that have experienced common respiratory infections (3). The alveolar macrophage phenotype changes in conjunction with improved lung defense, and these alveolar macrophages are essential when lungs with heterotypic immunity are challenged by high doses of bacteria. We postulate that such alveolar macrophage changes after repeated childhood infections may be integral parts of the many improvements in lung defense that render older children and young adults more resistant to pneumonia (3).

Other myeloid cells in the lung do not show differences in either number or surface marker phenotype, but further investigations would be necessary before concluding that such cells are unaffected by infection history. IMs or DCs (or IM or DC subsets) may change in experienced lungs in ways that could not be discerned using the limited panel of surface markers employed here. Studies should also address nonmyeloid cells of the lung (e.g., epithelial cells, endothelial cells, fibroblasts, pericytes, smooth muscle cells, neuronal cells, innate lymphocytes, and more) to see if they may phenotypically remodel after resolution of past infections.

Among the surface receptors we measured, SiglecF and MHC II are the most consistent markers of the altered phenotype. Surface SiglecF is consistently decreased due to experience, across all time points in an extended time course, in affected compared with contralateral lobes after localized infections, and due to spontaneous experiences that come with age in mice that did not receive experimental infections. Some studies consider decreased SiglecF to characterize alveolar macrophages that developed from monocytes recruited to the inflamed lung $(17,19)$. For the alveolar macrophages that remodel after adenoviral vector experience, SiglecF surface expression does not change (23), demonstrating a clear difference from the remodeling we observe after recovery from pneumococcal infection. Importantly, this highlights that different types of infectious history cause different types of alveolar macrophage remodeling. In the adenoviral vector studies, the remodeled alveolar macrophages are embryonic in origin, without contributions from bone marrow precursors (23); this is consistent with the concept that decreased SiglecF associates with cellular ontogeny. This implies that bone marrow-derived alveolar macrophages may be a component of the remodeling of lungs recovered from pneumococcal infection, as has been previously observed (30) and as supported by transcriptome analyses in the present study. During an inflammatory response, the autochthonous alveolar macrophages and the monocyte-derived macrophages recruited by that inflammation have very different transcriptomes and behaviors $(17,45)$. However, in the resting noninflamed lung, alveolar macrophages with these different cellular ontogenies are largely similar in their resting transcriptomes and responses to bacteria (19). The present studies reveal that the remodeled alveolar macrophages after pneumococcal infection have markedly different transcriptomes and metabolomes in the resting lung and distinct responses to bacterial infections. Thus, whether or not SiglecF is an indicator of varied ontogeny in lungs with resolved infections, cellular origin is insufficient to explain the remodeled alveolar macrophage phenotype after the resolution of pneumococcal pneumonia. 
The other surface marker phenotype that was especially pronounced and consistent in these studies, MHC II, was a defining feature of the alveolar macrophages that remodeled after adenoviral vector exposures (23). Although SiglecF changes differently in these 2 settings (decreased after pneumococcus but not after adenoviral vector), MHC II changes similarly (increased after either infection). Thus, increased MHC II surface expression may be a particularly robust marker of alveolar macrophages that have remodeled in response to infection history. The physiological significance of increased MHC II on remodeled alveolar macrophages is unknown. It will be of interest for future studies to determine whether and how the MHC II-expressing alveolar macrophages in experienced lungs present antigen to CD4+ $\mathrm{T}$ cells.

Metabolism is key for virtually all macrophage antimicrobial defenses (46). Creatine metabolism was a particularly prominent difference in experienced compared with naive alveolar macrophages across multiple experimental designs and analyses (Supplemental Figure 2). Phosphocreatine is a store of readily accessible, high-energy phosphates, and elevated phosphocreatine facilitates ATP regeneration to improve responses for fluctuating energy demands. In macrophages, phosphocreatine can serve as an energy source (47), and creatine kinase activities that generate phosphocreatine are necessary for optimal phagocytosis (48). Thus, elevated phosphocreatine could accelerate antimicrobial defense, typical of lungs with heterotypic immunity (11), by providing an increased source of energy for immune defenses, including phagocytosis. Upstream of phosphocreatine, creatine is an antioxidant that can help protect cells and tissues against oxidative damage (44). Intriguingly, creatine and the Slc6a8 creatine transporter that was strongly increased by prior pneumococcal exposures can directly influence polarized macrophage cytokine expression, favoring the "alternative" activation phenotype in bone marrow-derived macrophages, perhaps by modifying chromatin accessibility (49). The alveolar macrophages that remodeled after pneumococcal pneumonia displayed elevated creatine without a skewing toward "alternative" activation, but it is possible that creatine may influence chromatin accessibility and transcriptome changes in this setting as well. Increased Slc6a8 expression and elevated intracellular creatine also contribute to the efficacy of tumor-infiltrating $\mathrm{CD} 8^{+} \mathrm{T}$ cells, likely via energy metabolism (50). Together with our data, these observations suggest that enhancing creatine uptake may emerge as a broadly applicable means for immune cells to orient and augment their activities. Our metabolic and transcriptomic analyses highlighted creatine generation and import in alveolar macrophages remodeled after infection resolution, suggesting mechanisms by which creatine and phosphocreatine may be bolstered to empower hardier and healthier responses to subsequent infectious challenges.

The transcriptomic analyses reveal that alveolar macrophages with different infection histories not only have a new baseline but also respond distinctly to subsequent infection. At rest, experienced alveolar macrophages exhibit decreased cell cycle and mRNA processing activities, with no pathways reflecting a converse increase in activity. However, when lungs are acutely infected by a highly virulent pneumococcus, the alveolar macrophages that have recovered from prior infections again differ from naive counterparts, but in this case a distinct spectrum of activities are implicated and change in the opposite direction. The experienced lungs contain alveolar macrophages with no pathways identified to be decreased in activity, but multiple pathways are increased in activity, including immune signaling, glycoprotein processing, and energy metabolism. Alveolar macrophages in the experienced lung appear therefore to be more dynamic in their activity states, with quieter resting periods and more exuberant responses to an acute infectious challenge.

Alveolar macrophage transcriptome analyses showed that the mouse responses to pneumococcus are significantly concordant with human responses, and resolution of prior infections renders mouse alveolar macrophages even more adult human-like in their responses. The effect of prior experience with infections on similarities between species was small but in the right direction. The similarities are especially notable because incongruous experimental designs between the studies introduce many differences beyond the species difference of interest. The human studies involved alveolar macrophages cultured under highly controlled in vitro settings, while the mouse experiments involved alveolar macrophages in the more dynamic and much more physiologically complex setting of a living lung. The human studies involved alveolar macrophages from subjects with diverse and unknown variations throughout their genomes, throughout their infection histories (including the numbers, causes, and severities of prior infections), and throughout the rest of their environmental histories (including innumerable variables, such as age, diet, exercise, comorbidities, toxins, drugs, psychological stresses, sleep cycle, and microbiota). In contrast, the mouse experiments involved alveolar macrophages from an inbred strain, so study subjects had nearly identical genomes; environmental histories were designed to be as fully uniform as possible (other than differences introduced for the purpose of the experiment). Furthermore, the serotype 14 isolate of pneumococcus used as a stimulus 
in the human studies differed from the serotype 3 isolate used in the mouse studies. Despite these many experimental design differences, the bioinformatics analyses suggest that mouse and human alveolar macrophages respond quite similarly to pneumococcus, and a prior history of respiratory infections makes the mouse alveolar macrophage responses more humanlike.

The infections under study here were self-limited pneumococcal infections. They were not severe enough to require antibiotics or any other treatment, which represents a much milder and more limited infectious history than the typical human experiences. This setup is expected to simulate at best a small fraction of the immunological remodeling that occurs in humans, and the evidence supports that conclusion. Exposure of laboratory mice to mice purchased in pet stores is a much more intense infectious challenge (with laboratory mice seroconverting against many diverse pathogens, including bacteria, viruses, and parasites, and more than a fifth of those mice dying) and leads to more profound immunological remodeling (8). More controlled and lifelong exposures of laboratory mice to microbiota from wild mice extensively remodels leukocytes in the blood and spleen but not leukocytes in the vagina or skin (9), suggesting that infections of relevant tissues may be key. Practical ways to provide animals with relevant infection histories will help drive the next wave of immunological breakthroughs. Localized effects observed here (with alveolar macrophages in the infected but not the contralateral lobes remodeling after lobar pneumonia), which similarly apply to $\mathrm{CD}^{+}$Trm cells (11), indicate that the site of infection can be paramount. In addition, microbial factors, such as species and virulence properties, are important. As an example, alveolar macrophages have now been observed to remodel after resolution of influenza infections (15), adenoviral vector exposures (23), and pneumococcal infections (here), but how they remodel differs in each case. Surface SiglecF was decreased after pneumococcus but not adenovirus (23). Induction of CXCL1 and CXCL2 by bacterial stimuli was increased after adenovirus (23), decreased after influenza (15), and unaltered after pneumococcus. The types, numbers, and severities of infections in an animal's history must now be considered critical elements of experimental design and interpretation.

Alveolar macrophages remodel after resolution of pneumococcal pneumonia. The remodeled alveolar macrophages are quieter at rest, more active and effective when challenged, and more humanlike in their responses. Infection history is an important experimental design component that can and should be factored in to animal studies and their interpretations to elucidate the biological processes that determine how human lungs respond to pathogens. An improved understanding of how resolution of infection affects systemic and localized lung immunity will help translate findings between animal studies and human medicine, further guiding the development of immunomodulatory strategies designed to prevent or cure pneumonia and other infectious diseases.

\section{Methods}

A complete description of methods is provided in Supplemental Methods. Data sets from transcriptome studies were deposited into the National Center for Biotechnology Information's Gene Expression Omnibus database (ID GSE133975). Animal protocols were approved by the Boston University Institutional Animal Care and Use Committee.

\section{Author contributions}

AG and JPM conceived of and designed the experiments. AG, EIA, KAB, IM, ATS, AKW, CLDA, and JHE performed experiments. All authors discussed data and interpretations. ACB analyzed cytometry data. $\mathrm{HB}$ analyzed metabolome data. $\mathrm{AD}$ and $\mathrm{AL}$ performed comparisons of human and mouse data sets. AG and JPM constructed the manuscript and wrote initial drafts. AG, EIA, KAB, ACB, AL, HD, KET, MRJ, LJQ, and JPM revised the manuscript. JPM supervised the project.

\section{Acknowledgments}

Studies were supported by Bourse Mobilité (SRLF 2018) and the US NIH (R35 HL135756, R01 AI115053, R33 HL137081, F31 HL147397, F31 HL142199, R01 HL111459, R01 GM120060, R01 HL136725, K08 HL130582, UL1 TR001430, T32 HL007035). The authors thank the Boston University School of Medicine Flow Cytometry Core Facility for assistance with flow cytometry, the Metabolite Profiling Core Facility of the Whitehead Institute (particularly Caroline A. Lewis) for quantifying metabolites in cell samples, and the Boston University Microarray and Sequencing Resource Core Facility (particularly Yuriy Alekseyev and Adam Gower) for assistance with microarray analyses. 
Address correspondence to: Joseph P. Mizgerd, Boston University School of Medicine, 72 E. Concord St., Boston, Massachusetts 02118, USA. Phone: 617.358.1156; Email: jmizgerd@bu.edu.

1. Dickson RP, Erb-Downward JR, Martinez FJ, Huffnagle GB. The microbiome and the respiratory tract. Annu Rev Physiol. 2016;78:481-504.

2. Quinton LJ, Mizgerd JP. Dynamics of lung defense in pneumonia: resistance, resilience, and remodeling. Annu Rev Physiol. 2015;77:407-430.

3. Quinton LJ, Walkey AJ, Mizgerd JP. Integrative physiology of pneumonia. Physiol Rev. 2018;98(3):1417-1464.

4. Rudan I, Boschi-Pinto C, Biloglav Z, Mulholland K, Campbell H. Epidemiology and etiology of childhood pneumonia. Bull World Health Organ. 2008;86(5):408-416.

5. Yu H, Wier LM, Elixhauser A. Hospital stays for children, 2009: statistical brief \#118. In: Healthcare Cost and Utilization Project (HCUP) Statistical Briefs. Rockville, Maryland, USA: Agency for Healthcare Research and Quality; 2011.

6. Dela Cruz CS, et al. Future research directions in pneumonia. NHLBI Working Group Report. Am J Respir Crit Care Med. 2018;198(2):256-263.

7. Mizgerd JP, Skerrett SJ. Animal models of human pneumonia. Am J Physiol Lung Cell Mol Physiol. 2008;294(3):L387-L398.

8. Beura LK, et al. Normalizing the environment recapitulates adult human immune traits in laboratory mice. Nature. 2016;532(7600):512-516.

9. Rosshart SP, et al. Laboratory mice born to wild mice have natural microbiota and model human immune responses. Science. 2019;365(6452):eaaw4361.

10. Teijaro JR, Turner D, Pham Q, Wherry EJ, Lefrançois L, Farber DL. Cutting edge: Tissue-retentive lung memory CD4 T cells mediate optimal protection to respiratory virus infection. J Immunol. 2011;187(11):5510-5514.

11. Smith NM, et al. Regionally compartmentalized resident memory T cells mediate naturally acquired protection against pneumococcal pneumonia. Mucosal Immunol. 2018;11(1):220-235.

12. Allie SR, et al. The establishment of resident memory B cells in the lung requires local antigen encounter. Nat Immunol. 2019;20(1):97-108.

13. Liu Z, et al. Fate Mapping via Ms4a3-expression history traces monocyte-derived cells. Cell. 2019;178(6):1509-1525.e19.

14. Gomez Perdiguero E, et al. Tissue-resident macrophages originate from yolk-sac-derived erythro-myeloid progenitors. Nature. 2015;518(7540):547-551.

15. Didierlaurent A, et al. Sustained desensitization to bacterial Toll-like receptor ligands after resolution of respiratory influenza infection. J Exp Med. 2008;205(2):323-329.

16. Janssen WJ, et al. Fas determines differential fates of resident and recruited macrophages during resolution of acute lung injury. Am J Respir Crit Care Med. 2011;184(5):547-560.

17. Misharin AV, et al. Monocyte-derived alveolar macrophages drive lung fibrosis and persist in the lung over the life span. $J$ Exp Med. 2017;214(8):2387-2404.

18. Watanabe S, Alexander M, Misharin AV, Budinger GRS. The role of macrophages in the resolution of inflammation. J Clin Invest. 2019;129(7):2619-2628.

19. Gibbings SL, et al. Transcriptome analysis highlights the conserved difference between embryonic and postnatal-derived alveolar macrophages. Blood. 2015;126(11):1357-1366.

20. Netea MG, Schlitzer A, Placek K, Joosten LAB, Schultze JL. Innate and adaptive immune memory: an evolutionary continuum in the host's response to pathogens. Cell Host Microbe. 2019;25(1):13-26.

21. Bauer M, Weis S, Netea MG, Wetzker R. Remembering pathogen dose: long-term adaptation in innate immunity. Trends Immunol. 2018;39(6):438-445.

22. Domínguez-Andrés J, Joosten LA, Netea MG. Induction of innate immune memory: the role of cellular metabolism. Curr Opin Immunol. 2019;56:10-16.

23. Yao Y, et al. Induction of autonomous memory alveolar macrophages requires $\mathrm{T}$ cell help and is critical to trained immunity. Cell. 2018;175(6):1634-1650.e17.

24. Ramos-Sevillano E, Ercoli G, Brown JS. Mechanisms of naturally acquired immunity to Streptococcus pneumoniae. Front Immunol. 2019;10:358.

25. Jain S, et al. Community-acquired pneumonia requiring hospitalization among U.S. adults. NEngl J Med. 2015;373(5):415-427.

26. Jain S, et al. Community-acquired pneumonia requiring hospitalization among U.S. children. N Engl J Med. 2015;372(9):835-845

27. Pneumonia Etiology Research for Child Health (PERCH) Study Group. Causes of severe pneumonia requiring hospital admission in children without HIV infection from Africa and Asia: the PERCH multi-country case-control study. Lancet 2019;394(10200):757-779.

28. Coleman FT, et al. Capacity of pneumococci to activate macrophage nuclear factor $\kappa \mathrm{B}$ : influence on necroptosis and pneumonia severity. J Infect Dis. 2017;216(4):425-435

29. Dockrell DH, et al. Alveolar macrophage apoptosis contributes to pneumococcal clearance in a resolving model of pulmonary infection. J Immunol. 2003;171(10):5380-5388.

30. Taut K, et al. Macrophage turnover kinetics in the lungs of mice infected with Streptococcus pneumoniae. Am J Respir Cell Mol Biol. 2008;38(1):105-113.

31. Briles DE, et al. Immunizations with pneumococcal surface protein A and pneumolysin are protective against pneumonia in a murine model of pulmonary infection with Streptococcus pneumoniae. J Infect Dis. 2003;188(3):339-348.

32. Misharin AV, Morales-Nebreda L, Mutlu GM, Budinger GR, Perlman H. Flow cytometric analysis of macrophages and dendritic cell subsets in the mouse lung. Am J Respir Cell Mol Biol. 2013;49(4):503-510.

33. Levine JH, et al. Data-driven phenotypic dissection of AML reveals progenitor-like cells that correlate with prognosis. Cell. 2015;162(1):184-197. 
34. Belkina AC, Ciccolella CO, Anno R, Halpert R, Spidlen J, Snyder-Cappione JE. Automated optimized parameters for t-distributed stochastic neighbor embedding improve visualization and allow analysis of large data sets. Nat Commun. 2019;(10):5415.

35. Chakarov S, et al. Two distinct interstitial macrophage populations coexist across tissues in specific subtissular niches. Science. 2019;363(6432):eaau0964.

36. Gibbings SL, et al. Three unique interstitial macrophages in the murine lung at steady state. Am J Respir Cell Mol Biol. 2017;57(1):66-76.

37. Sichien D, Lambrecht BN, Guilliams M, Scott CL. Development of conventional dendritic cells: from common bone marrow progenitors to multiple subsets in peripheral tissues. Mucosal Immunol. 2017;10(4):831-844.

38. Dickson RP, Erb-Downward JR, Falkowski NR, Hunter EM, Ashley SL, Huffnagle GB. The lung microbiota of healthy mice are highly variable, cluster by environment, and reflect variation in baseline lung innate immunity. Am J Respir Crit Care Med. 2018;198(4):497-508.

39. Shenoy AT, et al. Lung $\mathrm{CD}^{+}{ }^{+}$resident memory $\mathrm{T}$ cells remodel epithelial responses to accelerate neutrophil recruitment during pneumonia [published online November 20, 2019]. Mucosal Immunol. https://doi.org/10.1038/s41385-019-0229-2.

40. Bewley MA, et al. Impaired mitochondrial microbicidal responses in chronic obstructive pulmonary disease macrophages. Am J Respir Crit Care Med. 2017;196(7):845-855.

41. Kamata H, et al. Epithelial cell-derived secreted and transmembrane 1a signals to activated neutrophils during pneumococcal pneumonia. Am J Respir Cell Mol Biol. 2016;55(3):407-418.

42. Murray PJ, et al. Macrophage activation and polarization: nomenclature and experimental guidelines. Immunity. 2014;41(1):14-20

43. Traber KE, et al. Induction of STAT3-dependent CXCL5 expression and neutrophil recruitment by oncostatin-M during pneumonia. Am J Respir Cell Mol Biol. 2015;53(4):479-488.

44. Joncquel-Chevalier Curt M, et al. Creatine biosynthesis and transport in health and disease. Biochimie. 2015;119:146-165.

45. Mould KJ, et al. Cell origin dictates programming of resident versus recruited macrophages during acute lung injury. $A m J$ Respir Cell Mol Biol. 2017;57(3):294-306.

46. Traven A, Naderer T. Central metabolic interactions of immune cells and microbes: prospects for defeating infections. $E M B O$ Rep. 2019;20(7):e47995.

47. Loike JD, Kozler VF, Silverstein SC. Increased ATP and creatine phosphate turnover in phagocytosing mouse peritoneal macrophages. J Biol Chem. 1979;254(19):9558-9564.

48. Kuiper JW, et al. Creatine kinase-mediated ATP supply fuels actin-based events in phagocytosis. PLoS Biol. 2008;6(3):e51.

49. Ji L, et al. Slc6a8-mediated creatine uptake and accumulation reprogram macrophage polarization via regulating cytokine responses. Immunity. 2019;51(2):272-284.e7.

50. Di Biase S, et al. Creatine uptake regulates CD8 T cell antitumor immunity. J Exp Med. 2019;216(12):2869-2882. 